\title{
A Comparison of Measured Temperatures with those Calculated Numerically and Analytically for an Exothermic Chemical Reaction inside a Spherical Batch Reactor with Natural Convection
}

\author{
A. N. Campbell ${ }^{*}$, S. S. S. Cardoso ${ }^{*}$ and A. N. Hayhurst \\ Department of Chemical Engineering, University of Cambridge, Pembroke Street, \\ Cambridge CB2 3RA, UK.
}

N.B. Figure 4 should occupy a whole page in landscape orientation.

${ }^{*}$ Corresponding Author. Tel. +44 1223 334777; Fax. +44 1223 334796;

E-mail addresses: anc31@cam.ac.uk, sssc1@cam.ac.uk 


\section{Abstract}

When any exo thermic chemical reaction occurs inside e.g. an unstirred spherical vessel, the heating effect of the reaction often induces temperature gradients and consequently natural convection. This work sets out to compare previously measured temperatures at different positions inside such a batch reactor with values computed numerically and analytically. It is the first such study for a reaction with an order greater than zero, occurring in a spherical reactor. The main reaction considered is the thermal decomposition of the gas, azomethane, which has often been used in experimental studies of thermal explosion. Other experimental results for the reaction between nitric oxide and oxygen, as well as between hydrogen and chlorine are also considered here. The measured temperatures at the centre of the vessel are first compared with analytical scales, derived by inspecting the governing equations. It is found that the temperature rise when diffusion is the dominant transport mechan ism (i.e. at small values of the Rayleigh number) is directly proportional to the ratio of the characteristic timescales for diffusion and for the reaction. Similarly, when natural convection is the dominant transport mechan ism, the temperature rise is proportional to the ratio of the timescales for convection and reaction.

A numerical scheme was developed to simulate the thermal decomposition of azomethane vapour under the influence of natural convection, as well as the diffusion of both matter and heat (via thermal conduction). The results of these simulations are compared with the temperature profiles measured along the vertical axis of the reactor. There is excellent agreement between experimental and numerical results. This confirms the computational procedures. The simulations indicate that the hottest point in the reactor moves upwards above the centre of the vessel when $R a$ is increased. In fact, three distinct types of temperature profile occur, depending on the value of $R a$. For low $R a$, the temperature profile is approximately spherically symmetric, as expected. When $R a$ is increased, the symmetry in the temperature profile is disrupted by the flow produced by natural convection. In that case, the temperature profile becomes skewed, with the maximum occurring on the axis in the top half of the reactor. Thirdly, under some circumstances at high $R a$, a large, sharp peak in the temperature profile near the top of the reactor is produced. This resembles a stabilised flame front, but could be an explosion. These three types of temperature distribution have also been observed experimentally. Finally, the ratio of the notional temperature rise in a purely diffusive system to that measured experimentally is compared over a wide range of $R a$. The experimental results for the three reactions considered all follow the same trend; the 
numerical and analytical solutions are in excellent agreement with the experimental results, confirming that the onset of natural convection inside a spherical vessel occurs at $R a \sim 500$. This result seems to be true, regardless of the order of the reaction, and only depends on the shape of the reactor.

Keywords: natural convection, mathematical modelling, simulation, fluid mechanics 


\section{Introduction}

When a gas undergoes an exothermic reaction within an unstirred batch reactor, spatial temperature gradients are induced. If these gradients become sufficiently large, the resulting buoyancy forces will cause the gas to move. This natural convection can have a significant influence on the progress of the reaction. The nature of the induced flow is determined by the Rayleigh number, $R a=\left(\beta g L^{3} \Delta T\right) /(\kappa v)$. In the classical work of Frank-Kamenetskii (1955) describing thermal explosion in a purely conductive system, it was suggested that in a gaseous system, natural convection becomes significant when $R a>10^{4}$. It should be noted that the condition actually states that natural convection will be significant when the Grashof number, $G r$, exceeds $10^{4}$. Since $R a$ is the product of $G r$ and the Prandtl number, and $\operatorname{Pr} \sim 1$ for a gas, then $R a$ and $G r$ are equivalent in this case. Further studies have shown that natural convection becomes important when $R a$ is at least an order of magnitude lower than this. Thus, experiments by Tyler (1966) and Ashmore et al. (1967) in the gas phase have demonstrated that in a spherical reactor, the effects of natural convection become evident when $R a$ rises above $\sim 600$. The experimental work of Merzhanov and Shtessel(1971) on liquid systems in a horizontal cylinder also indicated that natural convection has a significant influence at Rayleigh numbers well below the threshold value of $10^{4}$. These empirical observations of the effects of natural convection at lower values of $R a$ are supported by the analytical and numerical work of Jones (1973) and Shtessel et al. (1971); they showed that when a zeroth-order reaction occurred between parallel plates, the critical Rayleigh number for the onset of convection is again $\sim 500$. The assumption of zeroth-order kinetics in these previous theoretical studies is of course not a reflection of the true kinetics of the reactions considered, but an approximation. When the timescale during which the reaction is studied is much smaller than the timescale for the depletion of the reactant, the concentration of reactant decreases very little and may be assumed to remain constant, with a magnitude equal to the initial concentration. The assumption that the reactant has a constant concentration has been widely used in studies of natural convection coupled with an exothermic reaction. Jones (1973) also noted that inside a horizontal cylinder or a sphere, there is always some convection, because of the existence of temperature gradients perpendicular to the gravity vector; the 'onset' of convection is in fact merely the point where the effects of this flow become observable. Rayleigh numbers sufficiently large to induce significant natural convection are easily achievable in both experimental systems and industrial reactors. It is 
thus clear that natural convection can have a significant influence on the progress of an exothermic reaction.

This work is concerned with an exothermic reaction occurring in a spherical reactor. The walls of the reactor are kept at a constant temperature. Whilst reaction proceeds, heat is produced and consequently the temperature of the reacting gas rises, so that heat is removed from the system at the walls, which in fact represent an infinite heat sink compared to the finite amount of heat liberated by the reaction. The heating effect of the reaction and the removal of heat at the wall combine to create temperature gradients inside the reactor. In general, the temperature of the gas is at its maximum near the centre of the vessel and decreases radially towards the wall. The temperature gradients cause a gravitationally unstable distribution of density in the top section of the reactor and so leads to the development of the familiar Rayleigh-Bénard convection (Turner, 1979), shown schematically in Fig. 1. Part (a) shows the streamlines of the induced flow, and part (b) gives plots of both the temperature and density along the vertical axis of the reactor. The hot gas near the centre of the reactor rises quickly initially and moves into the hottest part of the reactor (in the top half). However, it slows after it passes through the hot zone, due to the decreased density difference and the proximity of the wall at the top of the vessel. The hot gas then contacts the comparatively cold walls, where it cools and descends relatively rapidly, due to the large difference in density between the cool gas at the wall and the much hotter gas near the centreline of the reactor. In the lower half of the reactor the density distribution is intrinsically stable, with the flow being induced by the descending, cooler gas at the wall. This downward flow of cool gas results in a relatively slow upward flow (around the vertical axis of the reactor) of gas displaced from the bottom of the reactor. Whilst this gas rises, it heats up and hence accelerates. The flow patterns typified by Fig. 1(a) mean that whilst the hottest part of the reactor is initially at the centre of the reactor, at longer times when the flow due to natural convection has developed, the hottest part of the reactor is significantly above the centre of the reactor, as shown schematically in Fig. 1(b). In many experimental studies, only the temperature at the centre of the reactor was measured; whenever natural convection is significant, th is temperature can be considerably lower than the maximum temperature achieved just above the centre.

The importance of taking account of natural convection in experimental work was shown by Gerri and Kaufman (1965), who studied the decomposition of azomethane vapour (at pressures up to $\sim 0.2$ bar) in a spherical vessel with the wall at a fixed temperature. An Arrhenius plot of their kinetic measurements showed curvature, indicating a change in 
apparent activation energy. Whilst non-linear Arrh enius plots can often be produced for so lid-state combustion reactions (e.g. Jones and Newman, 2003), they are not common in gas-phase systems over a modest range of temperature. Gerri and Kaufman (1965) speculated that this effect was due to a change in mechanism; however, Ashmore et al. (1967) showed that this change in apparent activation energy was consistent with natural convection developing in the reactor and skewing the temperature profile. Thus, an understanding of when natural convection becomes important, together with its magnitude and effects, is crucial for interpreting experimental work.

This work is concerned with comparing experimental results with numerical simulation and analytical scaling. Such comparisons have seldom been made previously. Merzhanov and Shtessel (1973) made a comparison of liquid phase systems in plane layers and cylinders. They studied the effect of natural convection on the critical value of the Frank-Kamenetskii parameter, $\delta_{c r}$, for explosion. This critical value indicates the transition from slow reaction to explosion in a purely diffusive system, such as would occur at very low pressures, in microgravity (where natural convection is suppressed by the absence of gravity) or in systems of reacting solids. The work of Merzhanov and Shtessel (1973) quantified how the transition to explosion is affected by natural convection, but did not provide any fundamental insight into the complex interaction of the flow due to natural convection and the potentially explosive chemistry.

Mitachi and co-workers (Mitachi et al., 1986; Mitachi and Igarashi, 1987) compared numerical simulations in a horizontal cylinder with experiments in which the exothermicity of the reaction was mimicked by electrical heating. In these comparisons only global average parameters, such as the average Nuss elt number for heat transfer, were compared. Mitachi and Igarashi (1987) used in terferometry to allow qualitative comparison of the spatial structure of the temperature fields generated experimentally and numerically; however, there has been very little quantitative comparison of the spatial development of e.g. the temperature field under the influence of natural convection when the reaction proceeds.

A further limitation of these few earlier comparisons of experimental and numerical work is that only pseudo-zeroth-order reactions were considered in the numerical studies. This ignores any spatial variation in the reaction rate due to the concentration varying throughout the reactor. Recent numerical studies of exothermic reactions occurring under the influence of natural convection (Campbell et al., 2005a, b; Campbell et al., 2006; Cardoso et al., 2004a, b) have considered a more complex reaction, namely that proposed by Sal'nikov 
(1949), consisting of two consecutive first-order steps. The concentration fields occurring during the course of this reaction are far more complex than those produced using the pseudo-zeroth-order approximation. Validation of the numerical simulations in these studies requires a comparison of experimental measurements with simulations, which include the effects of varying concentration on the rate of reaction.

In this paper, comparison will be made between previous exp erimental measurements and theoretical predictions, which are derived below in section 3. In addition, the experimental results will be compared with full numerical solutions of the governing equations in section 4. The experimental results considered are those of Archer (1977), who studied the thermal decomposition of azomethane vapour in a spherical reactor, and also those of Tyler (1966) and Ashmore et al. (1967), who studied the reactions of nitric oxide with oxygen, as well as chlorine with hydrogen, in a spherical reactor.

\section{Governing equations}

The first reaction considered is the thermal decomposition of azomethane $\left(\mathrm{CH}_{3} \mathrm{~N}_{2} \mathrm{CH}_{3}\right)$; the primary products are methane, ethane and nitrogen. Archer (1977) analysed the kinetics of the reaction at low pressures (up to $\sim 0.05$ bar) and found that the reaction had an order with respect to azomethane of $\sim 1.4$. Such a value means that the spatial variation of the rate of reaction, due to the concentration varying throughout the reactor, is likely to influence this system. Archer (1977) measured the rates of reaction over a temperature range of $612.2-$ $652.2 \mathrm{~K}$, with initially pure azomethane, as well as in the presence of diluents $\left(\mathrm{CO}_{2}\right.$ and $\left.\mathrm{SF}_{6}\right)$ and found the rate constant (for a reaction of order 1.4) can be written as:

$k / \mathrm{m}^{1.2} \mathrm{~mol}^{-0.4} \mathrm{~s}^{-1}=1.24 \times 10^{14} \exp \left(-194 \mathrm{~kJ} \mathrm{~mol}^{-1} / R T\right)$,

at the pressures of interest. The values of the activation energy, $E$, and the pre-exponential factor, $Z$, measured by Archer (1977) agree well with previous experimental studies (Camilleri et al., 1975; Riblett and Rubin, 1937; Rice, 1940; Trotman-Dickenson, 1955). The decomposition of azomethane was generally considered by these early workers to be quasiunimolecular. The reaction has been measured to be first-order at $\sim 0.4$ bar; however, Archer (1977) found the order to be 1.4 at lower pressures, i.e. neither at the high pressure limit of unity, nor at the low pressure limit of 2 . The non-integral order, between the low and high pressure limits indicates that at the pressures of interest, the reaction is complex. In the early studies the reaction was assumed simply to produce either, ethane and nitrogen, or two methyl radicals and nitrogen. The former is unlikely, given that the principal hydrocarbon 
product of the reaction is methane (Archer, 1977; Riblett and Rubin, 1937), which is likely to be produced by reaction of an azometh ane molecule with a methyl radical. Additional decomposition paths of e.g. $\mathrm{C}_{2} \mathrm{H}_{5} \mathrm{~N}_{2} \mathrm{CH}_{3}$ can explain the deviation from first-order kinetics measured by Archer (1977). Nevertheless, the order of 1.4 and Eq. (1), as measured by Archer (1977), are used below.

For the purposes of this work, the modelling approach first proposed by Cardoso et al. (2004b) is considered. The governing equations express the conservation of the reactants, energy, momentum and mass. These equations are simplified considerably by adopting the Boussinesq approximation, in which density changes are ignored, except insofar as they give rise to a buoyancy force; the conditions under which such a simplification is valid are discussed below. This simplified approach has been shown to produce temperature and concentration fields with spatial and temporal variations in qualitative agreement with experimental observations (Campbell et al., 2005a, b; 2006). More recently, it has been adopted and extended by others (Foster and Pearlman, 2006) in the modelling of cool flames. Below is presented the first quantitative comparison of th is modelling approach with experimental results.

The equation governing the conservation of the azomethane can be written as:

$\frac{\partial a}{\partial t}+\underline{u} . \nabla a=D_{A} \nabla^{2} a-k(T) a^{n}$,

where $a$ is the concentration of azomethane, $\underline{u}$ is the velocity vector, $D_{A}$ is the molecular diffusivity of azomethane and $n$ is the order of the reaction with respect to azomethane, taken here to be 1.4. The conservation of energy in the reactor is:

$\frac{C_{V}}{C_{P}} \frac{\partial T}{\partial t}+\underline{u} . \nabla T=\kappa \nabla^{2} T+\frac{q k(T)}{\rho_{0} C_{P}} a^{n}$,

where $C_{V}$ and $C_{P}$ are the mixture's specific heats at constant volume and pressure, $T$ is its temperature, $\kappa$ is the thermal diffusivity, $\rho_{0}$ is the density at the in itial temperature $T_{0}$ and $q$ is the exothermicity of the reaction. The familiar Navier-Stokes equations describe conservation of momentum:

$\frac{\partial \underline{u}}{\partial t}+\underline{u} . \nabla \underline{u}=-\frac{1}{\rho_{0}} \nabla\left(P-P_{0}\right)+v \nabla^{2} \underline{u}+\frac{\rho-\rho_{0}}{\rho_{0}} \underline{g}$,

where $P$ is the pressure in the reactor and $v$ is the kinematic viscosity. As mentioned above, the Boussinesq approximation is adopted, i.e. it is assumed that the density only varies in the buoyancy term of Eq. (4), in which $\rho=\rho_{0}\left[1-\beta\left(T-T_{0}\right)\right]$, where $\beta$ is the coefficient of thermal expansion. The density is assumed constant in all other terms in the governing 
equations. The Boussinesq approximation requires that the characteristic temperature rise $\Delta T$ is such that $\left(\Delta T / T_{0}\right)<<1$; otherwise full compressibility needs to be taken into account. Indeed, it can be seen below that for the majority of the reactions considered the ratio $(\Delta T /$ $T_{0}$ ) is of order 0.05 . This approximation is commonly used in the analysis of buoyant flows (Turner, 1979), and was universally used in all previous numerical studies of natural convection driven by an exothermic reaction. It should be noted that both the pressure and molar density inside the reactor will change due to one mole of azometh ane forming more than one mole of products. The molar ratio of the products of the reaction to the reactant has been measured at $\sim 2$ (Archer, 1977; Riblett and Rubin, 1937). This increase in the number of moles of gas in the reactor, of course, leads to an increase in pressure. This effect has been ignored in this work. This is because over the time period of interest (the first $10 \mathrm{~s}$ of reaction), the reaction only proceeds to $\sim 5 \%$ of completion (Archer, 1977), so the effect of the increased number of moles of gas due to reaction is slight. In addition, for many of the situations considered, a diluent is present in the reactor, thereby minimising the overall increase in the number of moles of gas inside the reactor and hence the consequential chan ges in pressure and density. The final equation required is the continuity equation. Adoption of the Boussinesq approximation allows the continuity equation to be written in its incompressible form, i.e.

$\nabla \cdot \underline{u}=0$

Initially, the reactor is considered to contain either pure azomethane, or a mixture of azomethane and a diluent. In the cases with a diluent, it is assumed that the gases are initially well-mixed. The gas is also assumed to be motionless initially, and at a uniform temperature $T_{0}$; this is the wall temperature, which remain s fixed at $T_{0}$ throughout the course of the reaction. This condition, of course, means that heat will be removed from the system at the wall. It is assumed that the no-slip condition applies at the wall and that there is no flux of any species at the wall. Consequently, the effects of any heterogeneous reactions at the wall have been ignored.

\section{Scaling Analys is}

\subsection{Development of Scales}

By examination of the governing equations (2) - (5), it is possible to derive analytical expressions describing the behaviour of certain process parameters, for example, the 
temperature rise due to reaction, and the velocity due to convection. The governing equations are first made dimensionless by introducing the following six dimension less variables:

$$
a^{\prime}=\frac{a}{a_{0}} ; T^{\prime}=\frac{T-T_{0}}{\Delta T} ; \underline{u^{\prime}}=\frac{\underline{u}}{U} ; P^{\prime}=\frac{P-P_{0}}{\rho_{0} U^{2}} ; \underline{x^{\prime}}=\frac{\underline{x}}{L} \text { and } t^{\prime}=\frac{U t}{L}, \quad(6 \mathrm{a}-\mathrm{f})
$$

where $a_{0}$ is the initial concentration of azomethane, $L$ is a characteristic length of the reactor, taken to be the radius in the present work, $\Delta T$ is the scale or characteristic value of the temperature rise and likewise $U$ is the characteristic magnitude of the velocity. At this stage the form of the scales for velocity and temperature rise are unknown. Using the variables defined in Eq. (6), Eqs. (2) - (5) become:

$$
\begin{aligned}
& \frac{\partial a^{\prime}}{\partial t^{\prime}}+\underline{u^{\prime}} \cdot \nabla^{\prime} a^{\prime}=\frac{D_{A}}{U L} \nabla^{\prime 2} a^{\prime}-\frac{k_{0} L a_{0}^{n-1}}{U} \exp \left(\frac{\phi T^{\prime}}{1+\eta T^{\prime}}\right) a^{\prime n}, \\
& \frac{C_{V}}{C_{P}} \frac{\partial T^{\prime}}{\partial t^{\prime}}+\underline{u}^{\prime} \cdot \nabla^{\prime} T^{\prime}=\frac{\kappa}{U L} \nabla^{\prime 2} T^{\prime}+\frac{q k_{0} L a_{0}^{n}}{\rho_{0} C_{P} U \Delta T} \exp \left(\frac{\phi T^{\prime}}{1+\eta T^{\prime}}\right) a^{\prime n}, \\
& \frac{\partial \underline{u^{\prime}}}{\partial t^{\prime}}+\underline{u^{\prime}} \cdot \nabla^{\prime} \underline{u^{\prime}}=-\nabla^{\prime} P^{\prime}+\frac{v}{U L} \nabla^{2} \underline{u}-\frac{L \beta \Delta T T^{\prime}}{U^{2}} \underline{g}, \\
& \nabla^{\prime} \cdot \underline{u^{\prime}}=0,
\end{aligned}
$$

where $k_{0}$ is the rate constant $k$ evaluated at the wall temperature, $T_{0}, \nabla^{\prime}$ is the dimensionless Laplacian operator and

$$
\eta=\frac{\Delta T}{T_{0}} \text { and } \phi=\frac{E \Delta T}{R T_{0}^{2}} \text {. }
$$

The transport of heat and mass within the reactor could be controlled by either diffusive or convective processes, depending on the value of $R a$. The magnitudes of the unknown scales will depend therefore on which of these mechanisms is dominant for the conditions prevailing in the reactor. These two regimes are examined below, in turn, to develop the most appropriate form for the unknown scales.

\subsubsection{Diffusion dominates transport}

For Rayleigh numbers much less than $\approx 500$, natural convection will be largely absent and diffusion will be the dominant mechanism for the transfer of mass; also, heat transfer occurs principally via thermal conduction. In this case the temperature and concentration fields are approximately spherically symmetric, with the maximum temperature occurring near the centre of the vessel. For these low Rayleigh numbers, Eqs. (9) and (10) for the conservation of momentum can be neglected, as can the convective terms on the left hand side of Eqs. (7) and (8). In such a diffusive system, the characteristic velocity, $U$, in Eqs. (7) 
and (8) is replaced by the ratio $\kappa / L$. A scale for $\Delta T$ can be found by assuming the terms for the conduction, and the generation of heat dominate Eq. (8). This yields

$$
\Delta T \sim \frac{q k_{0} L^{2} a_{0}^{n}}{\rho_{0} C_{P} \kappa} .
$$

It is useful at this stage to define characteristic timescales for diffusion and reaction as:

$$
\tau_{\text {diffusion }}=\frac{L^{2}}{\kappa} \text { and } \tau_{\text {reaction }}=\frac{\rho_{0}}{k_{0} a_{0}^{n}}
$$

These definitions can be substituted back into Eq. (12), which can be rearranged into dimensionless form giving

$$
\gamma \frac{\Delta T}{\Delta T_{a d}} \sim \frac{\tau_{d i f f u s i o n}}{\tau_{\text {reaction }}}
$$

where $\Delta T_{a d}$ is the adiabatic temperature rise $\left(=q / C_{V}\right)$ and $\gamma$ is the ratio of the specific heats.

\subsubsection{Natural convection dominates transport}

When the Rayleigh number rises well above $\approx 500$, natural convection becomes significant. The flow induced by the reaction distorts the spherical symmetry observed when diffusion dominates, and leads to the formation of a hot zone above the centre of the reactor (Cardoso et al., 2004a, b), as shown in Fig. 1(b). Now the buoyancy and convective terms dominate the Navier-Stokes equations (9); therefore the scale for the velocity can be defined as

$U \sim[\beta g L \Delta T]^{1 / 2}$.

Similarly, if it is assumed that the convection and generation terms dominate in the energy balance, Eq. (8), the scale for $\Delta T$ can be defined as

$$
\Delta T \sim \frac{q k_{0} a_{0}^{n} L}{U \rho_{0} C_{P}}
$$

and, if Eq. (15) is substituted into this expression, it yields

$$
\Delta T \sim\left(\frac{q k_{0} a_{0}^{n}}{\rho_{0} C_{P}}\right)^{2 / 3}\left(\frac{L}{\beta g}\right)^{1 / 3} .
$$

Equation (16) can be rewritten in dimensionless form by defining a timescale for natural convection as $\tau_{\text {convection }}=L / U$. Thus,

$\gamma \frac{\Delta T}{\Delta T_{\text {ad }}} \sim \frac{\tau_{\text {convection }}}{\tau_{\text {reaction }}}$,

which is interestingly similar to Eq. (14). 


\subsection{Comparison of Scaling with Experimental Results}

Archer (1977) measured the temperature at the centre of the reactor at the time when the temperature in the vessel was at its maximum. The forms of Eqs. (14) and (18) require estimates of the exothermicity of the reaction, $q$, and the specific heats for the contents of the reactor. Archer (1977) measured the exothermicity of the reaction, and found it to be weakly depend ent on the pressure, according to

$q=\frac{0.726}{P}+116.5$

where $q$ is in units of $\mathrm{kJ} \mathrm{mol}^{-1}$ and $P$ is in bar. This expression gives values for the heat of reaction in good agreement with those found elsewhere (Gerri and Kaufman, 1965; Rice, 1940). The fact that the exothermicity of the reaction depends on pressure suggests that changing the pressure alters the chemistry. Archer (1977) showed that, when azomethane decomposes, there was a large number of hydrocarbon products other than methane and ethane, mentioned above. Even a slight change in the balance of these products alters the heat of reaction. Nevertheless, over the range of pressures considered in this work, there is very little variation in $q$. Values of other physical parameters are shown in Table 1 . These values were aga in chosen to match those used by Archer (1977) and are in good agreement with literature values.

\subsubsection{Diffusion dominates transport}

Using the physical parameters defined previously, the experimentally measured temperatures can now be compared with the scales developed above. Figure 2 shows the measured dimensionless temperature rise (Archer, 1977) plotted against $\tau_{\text {diffusion }} / \tau_{\text {reaction }}$ at low Rayleigh numbers $(<100)$ for a range of values of $T_{0}$. In the experiments, $\tau_{d i f f u s i o n} /$ $\tau_{\text {reaction }}$ was varied by changing the initial concentration of azomethane in the reactor. All the results considered occurred in the region of slow reaction, i.e. below the explosive limit. Archer (1977) did perform experiments in the explosive regime; however, due to the large and very rapid increases in temperature associated with an explosion, the temperatures in the vessel were not monitored. It is clear from Fig. 2 that there are two different types of behaviour in the slow reaction regime. For very slow reaction, with low temperature rises, the linear scaling result in Eq. (14) is valid; however, beyond $\tau_{\text {diffision }} / \tau_{\text {reaction }} \sim 0.035$, there is a 
different regime, where the linear form of the scale breaks down. It is interesting to compare the point at which this change in regime occurs in the experimental results in Fig. 2 with the theoretical prediction of Frank-Kamenetskii (1955), for the transition from a slow reaction to an explosion. Frank-Kamenetskii (1955) showed that this transition depended only on a parameter $\delta$, which is a ratio of the initial rate of heat production by the reaction to the rate of heat loss by conduction. This parameter is defined as

$$
\delta=\frac{q E L^{2} k_{0} a_{0}^{n}}{\rho_{0} C_{P} \kappa R T_{0}^{2}} .
$$

If $\delta$ is less than a critical value, $\delta_{c r}$, which was found to be $\sim 3.32$ for a spherical vessel (Frank-Kamenetskii, 1955), there is a slow reaction, whereas if $\delta$ exceeds this critical value, there is an explosion. It should be noted that the form of $\delta$ proposed in Eq. (20) is slightly different to that initially defined by Frank-Kamenetskii (1955), which included a single constant to describe the rate of reaction. In Eq. (20), the rate of reaction is written explicitly as $k_{0} a_{0}^{n}$, showing the dependence of $\delta$ on the order of the kinetics and on the initial concentration of the reactant. Equation (20) can be rewritten in terms of the timescales defined in Eq. (13) as

$$
\delta=\frac{q E L^{2} k_{0} a_{0}^{n}}{\rho_{0} C_{P} \kappa R T_{0}^{2}}=\frac{E}{R T_{0}^{2}} \frac{q}{C_{P}} \frac{\tau_{\text {diffusion }}}{\tau_{\text {reaction }}} .
$$

Using this definition, and the critical value defined by Frank-Kamenetskii (1955), the value of $\tau_{\text {diffusion }} / \tau_{\text {reaction }}$ for the transition from slow reaction to explosion can be estimated. Shown in Fig. 2 are two vertical lines corresponding to the largest and smallest estimates of $\tau_{\text {difusion }} /$ $\tau_{\text {reaction }}$ for the transition to explosion, based on the physical conditions in Archer's (1977) experiments. A range of values of $\tau_{\text {diffusion }} / \tau_{\text {reaction }}$ for the transition from slow reaction to explosion is found because of uncertainties in the physical parameters in Eq. (21). The in itial temperature, $T_{0}$, affects the calculated value of $\tau_{\text {diffusion }} / \tau_{\text {reaction }}$, as does the initial pressure in the reactor, which has an impact on $q$ via Eq. (19). Clearly Fig. 2 indicates that the region in which $\delta$ exceeds the critical value for the onset of explosion corresponds to the change in the experimental results from the linear regime at small $\tau_{\text {diffusion }} / \tau_{\text {reaction }}$, to a non-linear regime at higher $\tau_{\text {diffusion }} / \tau_{\text {reaction }}$, below the experimental explosion limit. The scaling prediction in Eq. (14) is therefore valid in the very slow reaction regime of the reaction, but breaks down when the transition to what should be a thermal explosion approaches. The question of what occurs in the intermediate non-linear regime is considered below. 
For the linear regime, a numerical factor calculated using the least squares method can be introduced in Eq. (14) to give an expression for $\Delta T$ :

$\gamma \frac{\Delta T}{\Delta T_{a d}}=0.23 \frac{\tau_{\text {diffusion }}}{\tau_{\text {reaction }}}$

This scaling result for the slow reaction regime can be combined with Frank-Kamenetskii's (1955) parameter in Eq. (21) to give a condition for the transition from the linear regime to a non-lin ear one, below the transition to explosion, based on a temperature rise. Combining Eqs. (21) and (22) gives the condition that

$\Delta T<0.76 \frac{R T_{0}^{2}}{E}$,

for the linear form of the scale. Taking typical values for the azomethane system considered here, Eq. (23) shows that the linear regime occurs for $\Delta T<\sim 13 \mathrm{~K}$. Frank-Kamenetskii (1955) calculated that the transition from the non-linear regime to explosion occurs at:

$\Delta T=1.60 \frac{R T_{0}^{2}}{E}$,

for a spherical vessel. This gives a maximum pre-explosion temperature of $\sim 27 \mathrm{~K}$ for the gas-phase decomposition of azomethane. The maximum temperature rise measured below the explosive limit by Archer (1977) was $~ 33$ K, which agrees reasonably well with Eq. (24).

It is interesting to compare the experimental measurements for the decomposition of azomethane vapour presented by Archer (1977) with those performed for another exothermic reaction. Archer and Tyler (1976) measured temperatures at the centre of a spherical vessel during the thermal decomposition of 3-methyl-3-chlorodiazirine. These measurements were made at very low pressures ( $\sim 0.01$ bar $)$, so again natural convection was largely absent. The measurements of the temperature rises at two different wall temperatures showed exactly the same functional form as that shown in Fig. 2 for azomethane, when the pressure of 3-methyl3 -chlorodiazirine was increased. Thus, as $\tau_{\text {diffusion }} / \tau_{\text {reaction }}$ increased, the reaction exhibited first a linear increase in the temperature rise, followed by a non-linear regime and, fin ally, explosion occurred. Of course, the value of $\tau_{\text {diffusion }} / \tau_{\text {reaction }}$ at which the transition to explosion occurred is different to that for azomethane; the reason for this difference is evident in Eq. (21), which shows that $\delta$ depends on $E, q$ and $C_{P}$ in addition to $\tau_{\text {diffusion }} / \tau_{\text {reaction }}$. 


\subsubsection{Natural convection dominates transport}

A similar comparison can be made for the experimental results at higher Rayleigh numbers, because Archer (1977) also performed experiments at higher Rayleigh numbers, of $\sim 1000-2000$, i.e. when convection was significant. At such moderate Rayleigh numbers, diffusion would be expected to still play a role. As was the case above, only measurements in what was found to be the non-explosive regime are considered. Figure 3 shows the dimensionless temperature rise measured in the reactor plotted against $\tau_{\text {convection }} / \tau_{\text {reaction }}$; from Eq. (18), there should be a linear relationship. Once again there is excellent agreement between the experimental results and the form predicted by scaling. Equation (18) suggests that the experimental measurements should fall on a straight line through the origin in Fig. 3, and this is indeed the case. As with the diffusive regime, it is possible to include a numerical factor in the expression for the temperature rise. A least squares analysis indicates this can be written as:

$\gamma \frac{\Delta T}{\Delta T_{\text {ad }}}=2.97 \frac{\tau_{\text {convection }}}{\tau_{\text {reaction }}}$

\subsubsection{Comparison with scaling results for a different reaction scheme}

It is interesting to compare the results in Figs. 2 and 3 with another scaling analysis by Campbell et al. (2005a, 2006), for Sal'nikov's kinetic scheme, consisting of two, consecutive first-order reactions. In the purely diffusive case, it was shown by Campbell et al. (2005a, 2006) that the dimen sionless temperature rise for the slow reaction regime was also proportional to $\tau_{\text {diffusion }} / \tau_{\text {reaction }}$. The numerical coefficient calculated from the numerical results was of similar order to that derived above from the experimental measurements of Archer (1977). It is also interesting to note that the same deviation from linearity seen in Fig. 2, was also evident in the numerical results of Campbell et al. (2005a, 2006), where in that case, the deviation from linearity was due to the transition from the slow reaction regime, to an oscillatory regime with much larger temperature rises. The deviation from linearity was observed in simulations both with very weak natural convection and in the complete absence of convection. This deviation from linearity in the intermediate regime is due to the effects of the exponential term in Eq. (8). In the linear regime, the temperature rises are small enough for the exponential term to be $\sim 1$; however, when $\tau_{\text {diffusion }} / \tau_{\text {reaction }}$ is increased, the larger temperature rises that result, mean that the magnitude of the exponential increases and becomes significant, eventually leading to explosion. The agreement between the coefficients 
calculated in this work and that of Campbell et al. $(2005 \mathrm{a}, 2006)$ for the case when natural convection is significant is also rather good. The agreement for the two different reactions is expected, given that the constants should depend on the geometry of the system and not the order of the reaction.

\section{Comparison of Numerical Simulations with Experimental Results 4.1 Numerical method}

The above comparisons confirm the validity of the analytical scaling analysis. In addition, however, it is desirable to compare the results of numerical simulations with experimental observations. The governing equations (2) - (5) were solved numerically for a spherical reactor with a fixed wall temperature. The equations were solved using the PDE solver Fastflo (Fastflo Tutorial Guide, 2000), which utilises the finite element method. The algorithm used was similar to that outlined elsewhere (Cardoso et al., 2004b).

For the numerical simulations of the thermal decomposition of azomethane, the radius of the reactor was taken to be $L=0.064 \mathrm{~m}$, corresponding to a reactor with a volume of 1100

$\mathrm{dm}^{3}$, i.e. the size of vessel used in Archer's (1977) experiments. The order of the reaction was taken as 1.4 and the pre-exponential factor and activation energy were tho se derived by Archer (1977) in Eq. (1). The exothermicity of the reaction was calculated using Eq. (19). Various other physical parameters required estimation. These estimates were based on the initial conditions in the reactor and were assumed to remain constant. This assumption is made because the reaction only proceeds to $\sim 5 \%$ completion in the time simulated. The specific heat capacities were calculated using a simple weighted average of the components present. The thermal conductivity, and hence thermal diffusivity, of the gas mixture was calculated using Wassiljewa's equation with Mason and Saxena's modification (Reid et al., 1987). The viscosity of the gas mixture was estimated using Wilke's method (Reid et al., 1987). The molecular diffusivity of azomethane in the gas mixture was calculated using the correlation of Fuller et al., as suggested by Cussler (1984). Table 2 shows the physical parameters used in the numerical simulations. In addition, Table 2 shows both the simulated temperature rise at the centre of the reactor when the maximum temperature along the vertical axis of the reactor occurs, and the Rayleigh number, based on such a temperature rise at the centre. 


\subsection{Numerical results}

Numerical simulations were performed for the cases described in Table 2, with Rayleigh numbers in the range $13-21900$. As expected, the fields for the temperature and concentration of azomethane changed as $R a$ increased. Figure 4 shows the temporal evolution of the temperature and concentration fields within the reactor at three different Rayleigh numbers, which are typical of the different types of behaviour seen when $R a$ increases. Figure 4(a) corresponds to $R a=43$ in Table 2 . This is in the region where natural convection due to the heating effect of the reaction is very weak. Clearly, the temperature field shows the spherical symmetry expected whenever thermal conduction is the dominant mode of heat tran sfer. In addition, the radial temperature profile can be shown to be parabolic, as would be expected for a purely diffusive system. It should be noted that the temperature rise in this case is very small $(\sim 2 \mathrm{~K})$ due to the low initial concentration of azomethane, and hence the slow rate of reaction. The maximum temperature is reached relatively quickly, after $\sim 4 \mathrm{~s}$; subsequently, the rate of heat loss through the wall dominates the production of heat by the reaction, and hence the gas cools down. Inspection of the evolution of the concentration field in Fig. 4(a) shows that over the time period considered, the concentration of azomethane remains approximately spatially uniform. The decrease in azomethane concentration is very small $(\sim 7 \%)$ over the $10 \mathrm{~s}$ period of the simulation.

Figure 4(b) shows the development for $R a=1173$ in Table 2, in which natural convection is starting to play a role. The spherical symmetry evident in the temperature field at lower $R a$ has been disrupted by the flow due to natural convection. The toroidal vortex shown in Fig. 1(a) develops, causing the hottest point in the reactor to shift above the horizontal axis. As a result, the temperature profile along the vertical axis of the reactor becomes skewed. Once again, the concentration field remains virtually spatially uniform over the time period considered, and shows only a very small temporal decrease. There is some slight spatial inhomogeneity evident in the concentration of azomethane in Fig. 4(b) at $t=4.8$ $-5.6 \mathrm{~s}$. The concentration of azomethane in the top half of the reactor is marginally lower than in the surrounding fluid. This is unsurprising, given that this region correspond s to the hottest point in the reactor, and thus would have the largest rate of decomposition of azomethane. This effect is small, however, due to the relatively low maximum temperature rise.

As the temperature rise due to the reaction increases, natural convection becomes more intense (i.e. $R a$ increases) and the temperature field becomes much more skewed as a result. 
Under certain conditions, a distinct peak in the spatial variation of temperature emerges near the top of the reactor. This is shown in Fig. 4(c), which corresponds to $R a=21900$ in Table 2. The temperature peak, confined to the very top of the vessel due to the intensity of the natural convection, can very clearly be identified in Fig. 4(c). It is also evident that the temperature shows a very high degree of stratification, which is typical of a situation where natural convection is significant. The maximum temperature rise in this case is very large $(\sim 250 \mathrm{~K})$; however, this large increase in temperature is confined to a very small region of the reactor. The temperature distribution is therefore similar in form to a planar stabilised flame. In the bottom section of the vessel, the gas remains relatively cool and, in fact, the rises in temperature are an order of magnitude lower than those in the very hot region at the top of the reactor. The development of the concentration field is also far more complex in this case. It is clear from Fig. 4(c) that as the hot zone at the top of the reactor develops (at $t \sim 5 \mathrm{~s}$ ), the azomethane in this hot region is depleted very rapidly to virtually zero. As the reaction proceeds further, this depleted region grows, due to the influence of the toroidal flow pattern (Fig. 1(a)), and forms a horse-shoe shaped region in Fig 4(c) as the gas, which has been depleted of azomethane, flows downwards along the cool walls of the reactor. Meanwhile, at the bottom of the reactor the gas remains relatively cool, and hence is still somewhat rich in azomethane. This, of course, means that there are very large spatial variations in the concentration of azomethane inside the reactor; this is in contrast to the other two cases shown in Fig. 4, when the concentration remains approximately spatially uniform.

The three types of behaviour described above are in good qualitative agreement with the work of Archer (1977), whose experiments showed similar trends, i.e. a spherically symmetric temperature profile was observed at low $R a$, a skewed temperature profile, along the vertical axis of the reactor, at intermediate $R a$ and a peaked profile at high $R a$. This qualitative agreement is encouraging. Quantitative agreement was also sought, and this is now described below.

\subsection{Compariso $n$ of experimental and numerical resu lts}

\subsubsection{Comparing the measured and simulated temperature rises at the centre of the reactor}

Simulations were performed for the cases with $R a=13,43,54,97$ and 190 in Table 2. The simulated temperature rises at the cen tre of the reactor were then compared with the experimental results in Fig. 2. Shown in Fig. 5(a) are the experimental results in Fig. 2 for the diffusion-controlled regime, as well as a line representing the scaling result in Eq. (22). Also 
plotted are the results of the simulated cases mentioned above. There is clearly excellent agreement between the numerical simulations and both the experimental measurements, and the scaling analysis. A similar comparison is made for the case when natural convection is important in Fig. 5(b). In this case simulations were performed for $R a=701,936,1173$, 3451, 6130 and 9461; the results of these simulations appear in Fig. 5(b) along with the experimental measurements and scaling prediction (Eq. (25)). As was the case for the diffusive regime, the agreement is excellent.

\subsubsection{Comparing the shape of the measured and simulated temperature profiles}

In addition to comparing measured temperatures at the centre of the reactor with those found by simulation, it is also possible to compare the computed shapes of the temperature profiles in the reactor with those measured experimentally. As discussed above, when natural convection becomes vigorous, the maximum temperature in the vessel is found in the top half of the reactor. How far above the centre-line the maximum temperature occurs depends on the nature of the flow. Archer (1977) (using very fine thermocouples) measured the location of the maximum temperature along the vertical axis of the reactor and plotted this against the Rayleigh number, calculated using the temperature rise measured at the cen tre of the reactor at the same time as the maximum temperature along the vertical axis was observed. These measurements are reproduced in Fig. 6. Initially, for $R a<100$, the maximum temperature occurs very close to the centre of the reactor. When $R a$ increases beyond $\sim 300$ the position of the maximum temperature shifts upwards in the reactor. When $R a \sim 2000$, the maximum temperature is approximately halfway between the centre of the reactor and the wall at the top of the vessel. For further increases of $R a$, the position of the maximum temperature rises in the reactor. Above $R a \sim 10^{4}$, the location of the maximum temperature no longer moves, but remains at $\sim 0.8 \mathrm{~L}$ above the centre of the reactor. This upper limit is caused by the boundary layer at the wall, resulting from the wall being maintain ed at a lower temperature than the reactor's contents. Simulations of the cases shown in Table 2 were performed and the position of the maximum temperature for each established. These results are also plotted in Fig. 6, and the line for the best fit has been drawn through them. There is clearly very good agreement between the results of the simulations and Archer's (1977) measurements. At low $R a(<2000)$ the agreement is excellent, and at higher $R a$, although there is more of a discrepancy, the agreement is still very good, with the position of the final asymptote agreeing very well with the experimental results. For interest, the results of other numerical studies, but of a ps eudo-zeroth-order reaction occurring in a horizontal cylinder (Jones, 1974; 
Mitachi et al., 1986; Mitachi and Igarashi, 1987) have also been included. Because these studies consider a zeroth-order reaction, the temperature profiles described in Fig. 6 are the steady-state profiles. Although the geometry of the reactors is different, qualitatively the flow patterns in each in a circular cross-section through the vertical axis are similar. These numerical results are also in good agreement with the experimental observations of Archer (1977) and the simulations described in this work, both for a spherical reactor. This is unsurprising, because at low values of $R a$, the concentration of azomethane only decreases very slightly during the reaction, so it remains approximately spatially uniform, as shown in Fig. 4(a) and (b). This means that there is very little spatial variation of reaction rate due to spatial variations in concentration, so in that sense the reaction is behaving as if it were zeroth-order. However, at high values of $R a$, the numerical results for reaction in a cylinder move to the final asymptote of the position of the maximum rather gradually, whereas when azomethane reacts, this transition is more abrupt. The higher temperature rises at higher $R a$ result in the azomethane being depleted more rapidly in some parts of the reactor (Fig. 4(c)). This spatial variation is responsible for the deviation from the zeroth-order behaviour.

The three distinct regimes in Fig. 6 correspond to the cases shown in Fig. 4. There are the low and high $R a$ regimes where the location of the maximum temperature does not vary much when $R a$ is changed; also there is the intermediate regime where the position of the maximum temperature is very sensitive to $R a$. The temperature profile along the vertical axis of the reactor at the time when the maximum temperature occurs is plotted in Fig. 7 for the three regimes discussed above and considered in Fig. 4. As mentioned above, these three shapes for the temperature distribution were observed experimentally (Archer, 1977), as well as in the simulations. For low Rayleigh numbers $(<\sim 200)$ the temperature distribution is approximately symmetric, as shown in Fig. 7(a). This distribution is approximately parabolic, as described by Frank-Kamenetskii (1955). For the intermediate case $\left(200<R a<10^{4}\right)$, natural convection distorts the symmetry of the temperature distribution. Figure 7(b) shows a typical distribution in this region. The profile is skewed, with the highest temperature occurring above the cen tre of the reactor. The higher the Rayleigh number, the more skewed the profile. When $R a$ becomes sufficiently large, the hottest point in the reactor stops moving upwards. This is the third regime in Fig. 6, when, under certain conditions, a sharply peaked profile develops, as shown in Fig. 7(c). This type of profile typically, but not necess arily, emerges for $R a>10^{4}$. The shape of the temperature distribution in the bottom half of the reactor remains much the same as in the previous two cases. The temperature rise at the peak in Fig. $7(\mathrm{c})$ is high $(\sim 250 \mathrm{~K})$, because of the self-accelerating nature of the reaction in this 
part of the reactor. As mentioned above, the temperature profile in Fig. 7(c) is reminiscent of what would be observed in a stabilised flame. It is interesting to note that the upwards velocity of the gas near the vertical axis of the reactor reaches a maximum of $\sim 8 \mathrm{~cm} \mathrm{~s}^{-1}$ at the 'flame front'. This is similar in magnitude to the burning velocity of a decomposition flame of ethyl nitrate (Hicks, 1962), thereby confirming that this hot region has something of the character of a flame front. Archer (1977) observed experimentally that in virtually every case of such a peaked profile, the system proceeded to explosion. However, this effect was not observed in the simulations. The high temperature rises in the peak at the top of the vessel mean that the Boussinesq approximation (of constant den sity), and indeed other approximations (such as constant physical parameters) used in the numerical scheme described above, begin to break down. In fact, in the majority of the vessel the ratio $\left(\Delta T / T_{0}\right)$ remains below 0.1 , which is an acceptable level for applying the Boussinesq approximation. It is only in the very high temperature region in the top of the reactor, where $\left(\Delta T / T_{0}\right) \sim 0.4$, that the approximation breaks down. This must be borne in mind when examining the results; however, the simulation s do at least give a good qualitative insight into the behaviour of the reaction at these high temperatures. The transition to explosion, observed experimentally by Archer (1977), has not been considered further in this work.

\subsubsection{Comparing the asymmetry of the measured and simu lated vertical te mperat ure profiles}

As well as studying the effect of increasing $R a$ on the position of the maximum temperature within the reactor, Archer (1977) also examined the ratio of the temperature rises at different points along the vertical axis of the reactor. This gives a measure of the asymmetry of the temperature profile. Plotted in Fig. 8 are measurements expressed as ratios of the temperature rise at: (a) a distance $0.811 \mathrm{~L}$ above the cen tre of the reactor to the temperature rise at the centre $\left(\Delta T_{0}\right)$, and (b) a distance of $0.811 \mathrm{~L}$ below the centre of the reactor to the temperature rise at the centre. Once again, these ratios were calculated from the measured temperature profile at the time when the maximum temperature occurred in the reactor. The results of the numerical simulations shown in Table 2 are also plotted, with the best fit line drawn. The numerical studies for a horizontal cylinder (Jones, 1974; Mitachi et al., 1986; Mitachi and Igarashi, 1987) have also been included, where in these cases the temperature ratios were based on the steady-s tate temperature distribution. Figure 8(a) shows that for low $R a$ the ratio of the temperature near the top of the reactor and at the centre is $~$ 
0.3. Th is is the value expected for an approximately parabolic distribution, as in Fig. 7(a). When $R a$ increases above $\sim 1000$, the ratio begins to increase. There is good agreement between the experimental and numerical results in this region. Once $R a$ increases beyond 2000 the temperature ratio increases more rapidly above unity, until for $R a>10^{4}$ the peaked profile in Fig. 7(c) emerges, so the temperature near the top of the reactor is significantly larger than that at the centre of the reactor. Figure 8(a) shows this transition in both the experimental and numerical results. In fact, there is good agreement between the shapes of the experimental and numerical curves. Figure 8(b) shows a similar comparison for the bottom half of the reactor. It is immediately noticeable that the ratio of the temperature rise at $0.811 \mathrm{~L}$ below the centre of the reactor to the temperature rise at the centre of the reactor is independent of the Rayleigh number. Both experiments and numerics indicate that this ratio is constant at $\sim 0.3$. This indicates that the shape of the temperature profile in the bottom half of the reactor is largely independent of the intensity of the flow due to natural convection.

\subsubsection{Comparisons for $2 \mathrm{NO}+\mathrm{O}_{2} \rightarrow 2 \mathrm{NO}_{2}$}

Figure 8 gives an indication of how the temperature profile varies with $R a$, and shows that the numerical simulations are a good representation of what is observed experimentally in a spherical reactor. Unfortunately, no direct comparison can be made between the experimentally measured temperature profiles of Archer (1977) and those produced by the simulations in this work for the decomposition of azomethane, due to insufficient experimental measurements being available. However, Tyler (1966) and Ashmore et al. (1967) have measured the temperature profile for the different reaction:

$2 \mathrm{NO}+\mathrm{O}_{2} \rightarrow 2 \mathrm{NO}_{2}$,

occurring in a spherical vessel with nitrogen as a diluent. This reaction can be simulated, and the computed temperature profiles compared with the experimental results. The physical parameters were estimated using the methods described previously and the kinetics of the reaction are well established (Baulch et al., 1973); all these values appear in Table 3. Figure 9 shows the experimentally measured temperature profile $30 \mathrm{~s}$ after the commencement of reaction. This corresponds to the time at which Tyler (1966) and Ashmore et al. (1967) made their measurements. They describe the temperature increasing rapidly initially for $\sim 5 \mathrm{~s}$, before cooling down slowly. This qualitative description of the initial stages of the reaction matches what was found numerically. The situation shown in Fig. 9 represents a snapshot of the cooling period and corresponds to $R a \sim 8800$. Rayleigh numbers of this magnitude result 
in a skewed temperature profile. Also shown in Fig. 9 are the profiles of the maximum and minimum values found through simulation. These profiles were produced by taking the uncertainties in the estimates of the various physical parameters and the kinetics of the reaction in to account (Table 3 ). There is clearly excellent agreement between the experimental and numerical profiles. The shape of the profile achieved through simulation is very similar to that observed experimentally, with the position of the maximum temperature being the same in both cases. The quantitative agreement between the numerical and experimental results is also very good, with the experimental po ints lying between the maximum and minimum curves produced by simulation.

\subsubsection{Comparison of the experimental measurements for different reactions with the scaling and numerical results}

In addition to presenting the measured profile described above, Ashmore et al. (1967) also compared the temperature rise measured at the centre of the reactor, with that predicted by Frank-Kamenetskii (1955) for a purely conductive system, over a range of Rayleigh numbers. Frank-Kamenetskii showed that for a slow reaction below the explosive limit, the temperature rise at the centre of the reactor only depends on the value of the parameter $\delta$. The temperature rises corresponding to specific values of $\delta$ were calculated by solving the steady state energy balance (as in Eq. (8)) and were tabulated by Frank-Kamenetskii (1955). In the work of Ashmore et al. (1967), the Frank-Kamenetskii temperature rise was normalised by the observed temperature rise, and plotted against log $R a$. The plot presented by Ashmore et al. (1967) showed data for two different reactions occurring in a spherical reactor with a fixed wall temperature: (i) the reaction between nitric oxide and oxygen (Eq. (26)) in the presence of various diluents, and (ii) the reaction between hydrogen and chlorine. That plot is reproduced in Fig. 10. It should be noted that in the original plot (Ashmore et al., 1967), Ra was calculated using the Frank-Kamen etskii temperature rise; however, $R a$ in Fig. 10 has instead been calculated using the observed temperature rise at the centre of the reactor, at the time when the maximum temperature occurs in the vessel. Figure 10 very clearly shows the tran sition from diffusive control of transport, to a system where natural convection is dominant. This occurs at $R a \sim 500$. Ashmore et al. (1967) also showed that the experimental results of Gerri and Kaufman (1965) for the thermal decomposition of azomethane followed the same trend. These points are shown in Fig. 10, as well as the results of the numerical simulations in the present work described in Table 2. The values of the Frank-Kamenetskii 
temperature rise for the numerical cases were calculated by interpolating between the tabulated values presented by Frank-Kamenetskii (1955). There is good agreement between the results of the numerical simulations and the experimental studies of the various reactions. As well as comparing the numerics with these experimental results, it is also possible to compare the experiments with the scaling results described in section 3. The ordinate of Fig. 10 compares the temperature rise for a purely conductive system with the actual temperature increase. At low $R a$, when diffusion dominates transport, this ratio should be $\sim 1$. For higher Rayleigh numbers, when natural convection dominates transport, the scaling results, derived in Eqs. (22) and (25), suggest that:

$$
\frac{\Delta T_{\text {diffusion }}}{\Delta T_{\text {convection }}}=\frac{0.23 \frac{\tau_{\text {diffusion }}}{\tau_{\text {reaction }}}}{2.97 \frac{\tau_{\text {convection }}}{\tau_{\text {reaction }}}}=0.077 \frac{\tau_{\text {diffusion }}}{\tau_{\text {convection }}}=0.077(\mathrm{RaPr})^{1 / 2}
$$

The expression in Eq. (27) in fact over-pred icts the ratio of the Frank-Kamenetskii temperature rise to the observed temperature rise, shown in Fig. 10, by a factor of $\sim 1.5$. This discrepancy is most likely due to the difference between the Frank-Kamenetskii temperature rise, calculated for a zeroth-order system, and the scaling result derived in Eq. (22), confirmed by the experimental and numerical results for the non-zeroth order decomposition of azomethane. Nevertheless, the functional form in Eq. (27) is consistent with data presented in Fig. 10. Both the numerical and experimental results ind icate that the ratio of the diffusive temperature rise (calculated either using Frank-Kamenetskii's method or by Eq. (22)) to the observed temperature rise in a system where natural convection is important is proportional to $R a^{1 / 2}$, as shown by Eq. (27). Such agreement between the experimental results, the numerical simulations and the analytical scaling analysis provides an excellent validation of the theoretical approach in this work.

\section{Conclusions}

Comparisons have been made between experimental measurements of the temperature when an exothermic reaction occurs in a spherical vessel and the results of a scaling analysis of the governing equations, and also numerical simulation. It was found that for the two limiting cases where the transport of heat and mass within the reactor is controlled by diffusion or natural convection, the experimental results were consistent with the analytical scales developed, and the results derived also agreed well with the results of numerical simulations. It was found that the maximum temperature rise at the centre of the vessel is 
proportional to the ratio of the characteristic timescale for the dominant transport mechanism (i.e. diffusion or natural convection) and the timescale for reaction. This is similar to the results derived in a previous scaling analysis by Campbell et al. (2005a, 2006) for Sal'nikov's reaction scheme. The constants applied to the scaling expressions were found to be of similar order in both works. This is expected, since these constants should only depend on the geometry of the vessel. This confirms the generality and robustness of the approach developed here to describe these systems with natural convection and reaction.

The temperature profile along the vertical axis of the reactor measured experimentally was compared with the results of numerical simulation. Experiments showed that the position of the maximum temperature within the reactor moves up the axis as the Rayleigh number increases. This shift of the location of the maximum in temperature inside a reactor was also seen in the results of the numerical simulations, when the experimental and numerical results showed excellent agreement. In fact, the temperature profile measured experimentally for an exothermic reaction occurring in an unstirred spherical batch reactor agreed very well with that achieved through simulation, both in terms of the shape of the temperature distribution, and the magnitude of the temperature rise. Finally, a comparison of experimental results for three different exothermic reactions with both numerical and analytical results was made. The agreement between the three techniques proved to be excellent.

The experimental and numerical results presented showed that natural convection begin s to influence a system when $R a$ is in the range $100-1000$. The most sensitive indicator of when convection becomes important is given by the relationship between the position of the maximum temperature in the vessel along the vertical axis to $R a$. It has been shown that natural convection shifts this position above the centre of the reactor when $R a$ exceeds $\sim 100$; however, it is likely that the effects do not become significant until $R a$ increases above $\sim 500$, as shown in Fig. 10.

\section{Nomenclature}

$\begin{array}{ll}a & \text { concentration of azomethane } \\ a^{\prime} & \text { dimensionless concentration of azomethane, } a^{\prime}=a / a_{0} \\ a_{0} & \text { initial concentration of azomethane } \\ C_{P} & \text { specific heat at constant pressure } \\ C_{V} & \text { specific heat at constant volume } \\ D_{A} & \text { diffusion coefficient of azomethane }\end{array}$




\begin{tabular}{|c|c|}
\hline E & activation energy \\
\hline$g$ & acceleration due to gravity \\
\hline$G r$ & Grashof number, $G r=R a / P r$ \\
\hline$k_{T}$ & thermal conductivity \\
\hline$k_{i}$ & rate constant \\
\hline$k_{0}$ & rate constant evaluated at $T=T_{0}$ \\
\hline$L$ & characteristic length (radius) of the reactor \\
\hline$n$ & order of the reaction \\
\hline$P$ & pressure \\
\hline$P_{0}$ & initial pressure \\
\hline$P^{\prime}$ & dimensionless pressure, $P^{\prime}=P-P_{0} / \rho_{0} U^{2}$ \\
\hline$P_{i}$ & partial pressure of component $i$ \\
\hline $\operatorname{Pr}$ & Prandtl number, $\operatorname{Pr}=v / \kappa$ \\
\hline$q$ & exothermicity of the reaction \\
\hline$R$ & universal gas constant \\
\hline$R a$ & Rayleigh number, $R a=\beta g L^{3} \Delta T / \kappa v$ \\
\hline$t$ & time \\
\hline$t^{\prime}$ & dimensionless time, $t^{\prime}=U t / L$ \\
\hline$u$ & velocity \\
\hline$\underline{u}^{\prime}$ & dimensionless velocity, $\underline{u}^{\prime}=\underline{u} / U$ \\
\hline$U$ & characteristic velocity \\
\hline$T$ & temperature \\
\hline$T^{\prime}$ & dimensionless rise in temperature, $T^{\prime}=\left(T-T_{0}\right) / \Delta T$ \\
\hline$T_{0}$ & constant wall temperature \\
\hline$\underline{x}$ & spatial coordinates \\
\hline$\underline{x}^{\prime}$ & dimensionless spatial coordinates, $\underline{x}^{\prime}=\underline{x} / L$ \\
\hline$Z$ & pre-exponential factor in the Arrhenius term \\
\hline$\beta$ & coefficient of thermal expansion \\
\hline$\gamma$ & ratio of principal specific heats \\
\hline$\delta_{c r}$ & critical Frank-Kamenetskii parameter \\
\hline$\Delta T$ & characteristic temperature increase \\
\hline$\Delta T_{a d}$ & adiabatic temperature rise, $\Delta T_{a d}=q / C_{V}$ \\
\hline
\end{tabular}




$\begin{array}{ll}\eta & \text { dimensionless parameter, } \eta=\Delta T / T_{0} \\ \kappa & \text { thermal diffusivity } \\ \mu & \text { viscosity } \\ v & \text { kinematic visco sity } \\ \rho & \text { density } \\ \rho_{0} & \text { density at } T=T_{0} \\ \tau_{\text {convection }} & \text { timescale for convection } \\ \tau_{\text {diffusion }} & \text { timescale for diffusion of heat } \\ \tau_{\text {reaction }} & \text { timescale for the reaction } \\ \phi & \text { dimensionless activation energy, } \phi=E \Delta T / R T_{0}^{2}\end{array}$

\section{Acknowle dgements}

The financial support of the Engineering and Physical Sciences Research Council is gratefully acknowledged. We are also very grateful to Dr. B. J. Tyler for pointing us towards the experimental data used in this paper.

\section{References}

Archer, W.H. (1977). Heat transfer mechanisms in exothermic reactions. Ph.D. Thesis, University of Manchester Institute of Science and Technology.

Archer, W.H., Tyler, B.J. (1976). Self-heating in the decomposition of 3-methyl-3chlorodiazirin e: determination of reaction exothermicity and correction of Arrhenius parameters. Journal of the Chemical Society - Faraday Transactions I, 72, 1448 - 1455.

Ashmore, P.G., Tyler, B.J., Wesley, T.A.B. (1967). Experimental investigations of conductive and convective heat transfer in relation to thermal ignitions. Eleventh Symposium (International) on Combustion. The Combustion Institute, Pittsburgh, PA, pp. 1133-1140.

Baulch, D.L., Drysdale, D.D., Horne, D.G., Lloyd, A.C. (1973). Evaluated Kinetic Data for High Temperature Reactions, Vol. 2, Butterworths, London, pp. 285 - 300. 
Camilleri, P., Marshall, R.M., Purnell, H. (1975). Arrhenius parameters for the unimolecular decompositions of azomethane and n-propyl and isopropyl radicals and for methyl radical attack on propane. Journal of the Chemical Society, Faraday Transactions 1, 71, 1491 1502.

Campbell A.N., Cardoso S.S.S., Hayhurst A.N. (2005a). A scaling analysis of Sal'nikov's reaction, $\mathrm{P} \rightarrow \mathrm{A} \rightarrow \mathrm{B}$, in the presence of natural convection and the diffusion of heat and matter. Proceedings of the Royal Society A, 461, 1999 - 2020.

Campbell A.N., Cardoso S.S.S., Hayhurst A.N. (2005b). The influence of natural convection on the temporal development of the temperature and concentration fields for Sal'nikov's reaction, $\mathrm{P} \rightarrow \mathrm{A} \rightarrow \mathrm{B}$, occurring batchwise in the gas phase in a closed vessel. Chemical Engineering Science, 60, 5705 - 5717.

Campbell A.N., Cardoso S.S.S., Hayhurst A.N. (2006). A scaling analysis of the effects of natural convection, when Sal'nikov's reaction: $\mathrm{P} \rightarrow \mathrm{A} \rightarrow \mathrm{B}$ occurs, together with diffusion and heat transfer in a batch reactor. Chemical Engineering Research and Design, 84(A7), 553 $-561$.

Cardoso, S.S.S., Kan, P.C., Savjani, K.K., Hayhurst, A.N., Griffiths, J.F. (2004a). The computation of the velocity, concentration and temperature fields during a gas-phase oscillatory reaction in a closed vessel with natural convection. Combustion and Flame, 136, $241-245$.

Cardoso, S.S.S., Kan, P.C., Savjani, K.K., Hayhurst, A.N., Griffiths, J.F. (2004b). The effect of natural convection on the gas-phase Sal'nikov reaction in a closed vessel. Physical Chemistry Chemical Physics, 6, 1687 - 1696.

Cussler, E.L. (1984). Diffusion: Mass Transfer in Fluid Systems, Cambrid ge University Press, Cambridge, pp. $112-113$.

Fastflo Tutorial Guide, (version 3) 2000, July 1999 (online), CSIRO, Australia. 
Frank-Kamenetskii, D.A. (1955). Diffusion and Heat Exchange in Chemical Kinetics (translated by N. Thon). Princeton University Press, Princeton, pp. $202-266$.

Foster, M., Pearlman, H. (2006). Cool flames at terrestrial, partial, and near-zero gravity. Combustion and Flame, 147, 108 - 117.

Gerri, N.J., Kaufman, F. (1965). The explosive decomposition of azomethane. Tenth Symposium (International) on Combustion. The Combustion Institute, Pittsburgh, PA, pp. $227-235$.

Hicks, J.A. (1962). The low-pressure decomposition flame of ethyl nitrate. Eighth Symposium (International) on Combustion. The Combustion Institu te, Pittsburgh, PA, pp. 487 - 496.

Jones, D.R. (1973). The dynamic stability of confined, exothermically reacting flu ids. International Journal of Heat and Mass Transfer, 16, 157 - 167.

Jones, D.R. (1974). Convective effects in enclosed, exo thermically reacting gases. International Journal of Heat and Mass Transfer, 17, 11 - 21.

Jones, J.C., Newman, S.C. (2003). Non-Arrhenius behaviour in the oxidation of two carbonaceous substrates. Journal of Loss Prevention in the Process Industries, 16, 223 - 225.

Merzhanov, A.G., Shtessel, E.A. (1971). Thermal explosion in reacting liquid systems in the presence of thermal convection. Combustion, Explosion and Shock Waves, 7, 58 - 65.

Merzhanov, A.G., Shtessel, E.A. (1973). Free convection and thermal explosion in reactive systems. Astronautica ACTA, 18, 191-199.

Mitachi, K., Aoki, K., Kitamura, K., Komatsu, N. (1986). Natural convection of heat generating fluid within horizontal cylind er. Bulletin of JSME, 29, 2547- 2553.

Mitachi, K., Igarashi, M. (1987). Unsteady natural convection of heat-generating fluids in a horizontal cylin der. JSME International Journal, 30, 1099 - 1105. 
Reid, R.C., Prausnitz, J.M., Poling, B.E. (1987). The Properties of Gases and Liquids, $4^{\text {th }}$ edition, Mcgraw-Hill, New York, pp. $404-417$ \& pp. 530-535.

Riblett, E.W., Rubin, L.C. (1937). The thermal decomposition of azomethane. Journal of the American Chemical Society, 59, 1537 - 1540.

Rice, O.K. (1940). The role of heat conduction in thermal gaseous explosions. Journal of Chemical Physics, 8, $727-733$.

Sal'nikov, I.E., 1949. Contribution to the theory of the periodic homogeneous chemical reactions. Zhurnal Fizicheskoi Khimii, 23, 258 - 272.

Shtessel, E.A., Priytkova, K.V., Merzhanov, A.G. (1971). Numerical solution to the problem of a thermal explosion taking account of free convection. Combustion, Explosion and Shock Waves, 7, $137-146$.

Trotman-Dickenson, A.F. (1955). Gas Kinetics: an introduction to the Kinetics of Homogeneous Gas Reactions, Butterworths Scientific Publications, London, pp. 69 - 71.

Turner, J.S. (1979). Buoyancy Effects in Fluids. Cambridge University Press, Cambridge, pp. $207-250$.

Tyler, B.J. (1966). An experimental investigation of conductive and convective heat transfer during exothermic gas phase reactions. Combustion and Flame, 10, 90 - 91. 


\section{Table Captions}

Table 1. Physical parameter estimates for azomethane, $\mathrm{CO}_{2}$ and $\mathrm{SF}_{6}$. These estimates are taken from the work of Archer (1977).

Table 2. Details of the conditions in the reactor used in the numerical simulations. Also shown are the simulated temperature rise at the centre of the reactor and the corresponding Rayleigh number.

Table 3. Physical parameters used for the simulation of the reaction between nitric oxide and oxygen, the results of which appear in Fig. 9. 


\section{Figure Captions}

Fig 1. (a) Streamlines of the flow due to natural convection in a vertical cross section through the axis of the reactor. The toroidal vortex typifies the flow: upwards near the axis, downwards near the wall. (b) Temperature and density profiles along the vertical axis of the reactor, showing the unstable density distribution in the top half of the reactor and the stable density distribution in the bottom half of the reactor, where flow is driven by conditions in the boundary layers.

Fig. 2. Plot of the dimensionless temperature rise at the centre of the reactor against $\tau_{\text {diffusion }} /$ $\tau_{\text {reaction }}$ for the experimental results of Archer (1977) when diffusion controls transport. Initially, the reactor contained pure azomethane. Results are shown for different values of the wall temperature, $T_{0}$. Also shown are two vertical lines representing the approximate transition between slow reaction and explosion, as indicated by Frank-Kamenetskii's (1955) criterion, and also a line representing the scaling result in Eq. (22).

Fig. 3. Plot of the dimensionless temperature rise at the centre of the reactor against $\tau_{\text {convection }} /$ $\tau_{\text {reaction }}$ for the experimental results of Archer (1977) when natural convection controls tran sport. The reactor initially contained azomethane (Azm) and a diluent $\left(\mathrm{CO}_{2}\right.$ or $\left.\mathrm{SF}_{6}\right)$. Results are also shown for different values of $T_{0}$. The best-fit line shown corresponds to Eq. (25), derived through scaling.

Fig. 4. Evolution of the temperature field (top) and concentration (of azomethane) field (bottom) over the period $t=0-9.6 \mathrm{~s}$. The frames occur at regular intervals of $0.8 \mathrm{~s}$. Three cases are shown, corresponding to different values of $R a$. The details of the cases considered are given in Table 2, where (a) shows the $R a=43$ case, (b) shows the $R a=1173$ case and (c) shows the $R a=21900$ case. The temperature and concentration scales appear to the right and it should be noted that these are different for each case presented.

Fig. 5. (a) Plot of the dimensionless temperature rise at the centre of the reactor against

$\tau_{\text {diffusion }} / \tau_{\text {reaction }}$ for the experimental results of Archer (1977) when diffusion controls tran sport and the numerical results for the cases with $R a=13,43,54,97$ and 190 in Table 2. 
(b) Plot of the dimensionless temperature rise at the centre of the reactor against $\tau_{\text {convection }} /$ $\tau_{\text {reaction }}$ for the experimental results of Archer (1977) when natural convection controls transport and the numerical results for the cases with $R a=701,936,1173,3451,6130$ and 9461 in Table 2.

Fig. 6. Plot of the dimensionless position of the maximum temperature on the vertical axis of the reactor, for the thermal decomposition of azomethane. The experimental results of Archer (1977) are shown, as are the results of the numerical simulations. The line is a best fit through the numerical results. Also shown are the results of previous numerical studies (Jones, 1974; Mitachi et al., 1986; Mitachi and Igarashi, 1987) for a pseudo-zeroth-order reaction occurring in a horizontal cylinder.

Fig. 7. (a) Approximately symmetric temperature profile, derived through simulation, along the vertical axis of the reactor for a low $R a$, actually for $R a=43$ in Table 2. (b) Skewed temperature profile, for intermediate $R a$, showing the maximum temperature occurring above the centre of the reactor. Here, $R a=1173$. (c) Peaked profile, characteristic of a large $R a=$ 21900.

Fig. 8. (a) Ratio of the temperature rise at $0.811 \mathrm{~L}$ above the centre of the spherical reactor to the temperature rise at the centre plotted against $R a$. The exp erimental measurements of Archer (1977) are shown, as are the results of numerical simulations in Table 2. The line is drawn through the results of these simulations. Also shown are the results of previous numerical studies for a pseudo-zeroth-order reaction occurring in a horizontal cylinder. (b) Ratio of the temperature rise at $0.811 \mathrm{~L}$ below the cen tre of the reactor to the temperature rise at the centre plotted against $R a$.

Fig. 9. Comparison of the temperature profile measured by Tyler (1966), $30 \mathrm{~s}$ after the commencement of the reaction between nitric oxide and oxygen in the presence of nitrogen, with the results of numerical simulation. The reaction occurs in a spherical vessel (volume $1100 \mathrm{dm}^{3}$ ), with the wall held at $343 \mathrm{~K}$. There was initially $9330 \mathrm{~Pa}$ of $\mathrm{NO}, 4665 \mathrm{~Pa}$ of $\mathrm{O}_{2}$ and $12662 \mathrm{~Pa}$ of $\mathrm{N}_{2}$. The lines show the maximum and minimum profiles calculated numerically, based on the errors in the physical parameters. 
Fig. 10. The ratio of the temperature rise in a purely conductive system (calculated using Frank-Kamen etskii's method) to the observed temperature rise plotted against log $R a$. The experimental results of Ashmore et al. (1967) for two different exothermic reactions (NO with $\mathrm{O}_{2}$ and $\mathrm{H}_{2}$ with $\mathrm{Cl}_{2}$ ) occurring in a spherical reactor are shown, as are the experimental results of Gerri and Kaufman (1965) for the decomposition of azomethane. The results of numerical simulations for the thermal decomposition of azomethane are shown. Best fit curves through the numerical points are also shown. 
Table 1

\begin{tabular}{cccc}
\hline$T / \mathrm{K}$ & $k_{T} / \mathrm{W} \mathrm{m}^{-1} \mathrm{~K}^{-1}$ & $\mu \times 10^{5} / \mathrm{Pa} \mathrm{s}$ & $C_{V} / \mathrm{J} \mathrm{mol}^{-1} \mathrm{~K}^{-1}$ \\
\hline \multicolumn{3}{c}{ Azomethane } \\
612.2 & 0.0498 & 1.70 & 120.9 \\
616.2 & 0.0502 & 1.71 & 121.3 \\
626.2 & 0.0515 & 1.73 & 122.6 \\
636.2 & 0.0531 & 1.75 & 123.8 \\
646.2 & 0.0544 & 1.77 & 125.1 \\
652.2 & 0.0552 & 1.78 & 125.9 \\
636.2 & 0.0431 & $\mathrm{CO}_{2}$ \\
& \multicolumn{3}{c}{2.84} \\
626.2 & 0.0353 & $\mathrm{SF}_{6}$ \\
636.2 & 0.0360 & 2.91 & 39.6 \\
\hline
\end{tabular}

Table 2

\begin{tabular}{ccccccccc}
\hline$T_{0} / \mathrm{K}$ & $\begin{array}{c}P_{\text {azomethane }} / \\
\mathrm{Pa}\end{array}$ & $\begin{array}{c}P_{\mathrm{CO} 2} / \\
\mathrm{Pa}\end{array}$ & $\begin{array}{c}P_{S F \sigma} / \\
\mathrm{Pa}\end{array}$ & $\begin{array}{c}\kappa \times 10^{4} / \\
\mathrm{m}^{2} \mathrm{~s}^{-1}\end{array}$ & $\begin{array}{c}\mu \times 10^{5} / \\
\mathrm{Pa} \mathrm{s}\end{array}$ & $\begin{array}{c}D_{A} \times 10^{4} / \\
\mathrm{m}^{2} \mathrm{~s}^{-1}\end{array}$ & $\begin{array}{c}\Delta T_{\text {centre }} / \\
\mathrm{K}\end{array}$ & $R a$ \\
\hline 636.2 & 1333 & 0 & 0 & 16.0 & 1.75 & 19.4 & 6.2 & 13 \\
636.2 & 683 & 8626 & 0 & 4.62 & 2.72 & 3.76 & 1.7 & 43 \\
636.2 & 1973 & 0 & 0 & 10.8 & 1.75 & 13.1 & 11.7 & 54 \\
636.2 & 2266 & 0 & 0 & 9.38 & 1.75 & 11.4 & 15.8 & 97 \\
636.2 & 1029 & 13159 & 0 & 3.03 & 2.72 & 2.47 & 3.2 & 190 \\
636.2 & 1733 & 0 & 4666 & 2.47 & 2.71 & 3.17 & 7.8 & 701 \\
636.2 & 1733 & 20932 & 0 & 1.89 & 2.71 & 1.54 & 6.1 & 936 \\
636.2 & 1760 & 0 & 6786 & 1.80 & 2.77 & 2.37 & 7.0 & 1173 \\
626.2 & 4960 & 0 & 4960 & 1.71 & 2.44 & 1.99 & 15.3 & 2861 \\
636.2 & 2000 & 0 & 13332 & 0.964 & 2.84 & 1.32 & 6.0 & 3451 \\
636.2 & 2000 & 0 & 19998 & 0.658 & 2.88 & 0.922 & 5.0 & 6130 \\
636.2 & 3720 & 0 & 14399 & 0.847 & 2.77 & 1.12 & 11.5 & 8670 \\
636.2 & 4333 & 0 & 12346 & 0.944 & 2.72 & 1.22 & 15.1 & 9223 \\
636.2 & 2066 & 0 & 26664 & 0.499 & 2.89 & 0.706 & 4.4 & 9461 \\
636.2 & 3720 & 0 & 17332 & 0.718 & 2.80 & 0.964 & 10.5 & 10967 \\
636.2 & 4000 & 0 & 15999 & 0.765 & 2.78 & 1.01 & 11.9 & 10992 \\
636.2 & 4666 & 0 & 13332 & 0.875 & 2.72 & 1.13 & 16.2 & 11492 \\
636.2 & 5333 & 0 & 13332 & 0.854 & 2.70 & 1.09 & 20.1 & 15053 \\
636.2 & 6000 & 0 & 13999 & 0.803 & 2.68 & 1.01 & 25.8 & 21900 \\
\hline
\end{tabular}

Table 3

\begin{tabular}{ccc}
\hline parameter & estimate & units \\
\hline$C_{V}$ & $790 \pm 40$ & $\mathrm{~J} \mathrm{~kg}^{-1} \mathrm{~K}^{-1}$ \\
$C_{P}$ & $1100 \pm 50$ & $\mathrm{~J} \mathrm{~kg}^{-1} \mathrm{~K}^{-1}$ \\
$q$ & $113800 \pm 1200$ & $\mathrm{~J} \mathrm{~mol}^{-1}$ \\
$Z$ & $0.0024 \pm 0.0005$ & $\mathrm{~m}^{6} \mathrm{~mol}^{-2} \mathrm{~s}^{-1}$ \\
$E$ & $-4380 \pm 840$ & $\mathrm{~J} \mathrm{~mol}^{-1}$ \\
$\kappa$ & $(9.50 \pm 1.43) \times 10^{-5}$ & $\mathrm{~m}^{2} \mathrm{~s}^{-1}$ \\
$\mu$ & $(2.30 \pm 0.35) \times 10^{-5}$ & $\mathrm{~Pa} \mathrm{~s}^{-4}$ \\
$D_{N O, \text { mixture }}$ & $(1.10 \pm 0.22) \times 10^{-4}$ & $\mathrm{~m}^{2} \mathrm{~s}^{-1}$ \\
$D_{O 2, \text { mixture }}$ & $(1.05 \pm 0.21) \times 10^{-4}$ & $\mathrm{~m}^{2} \mathrm{~s}^{-1}$ \\
\hline
\end{tabular}




\section{Figure 1}

Click here to download high resolution image
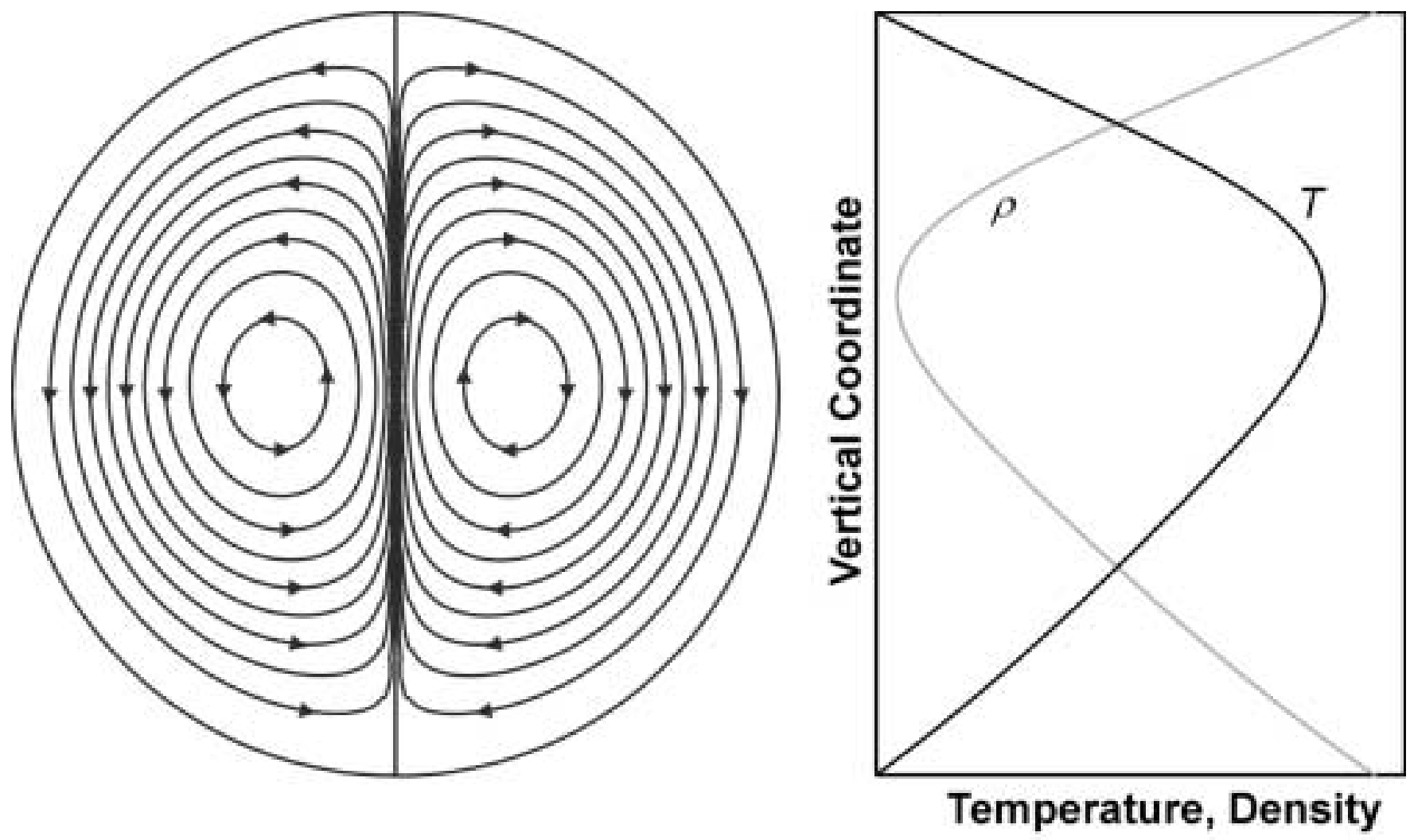

(a)

(b) 
Figure 2
Click here to download high resolution image

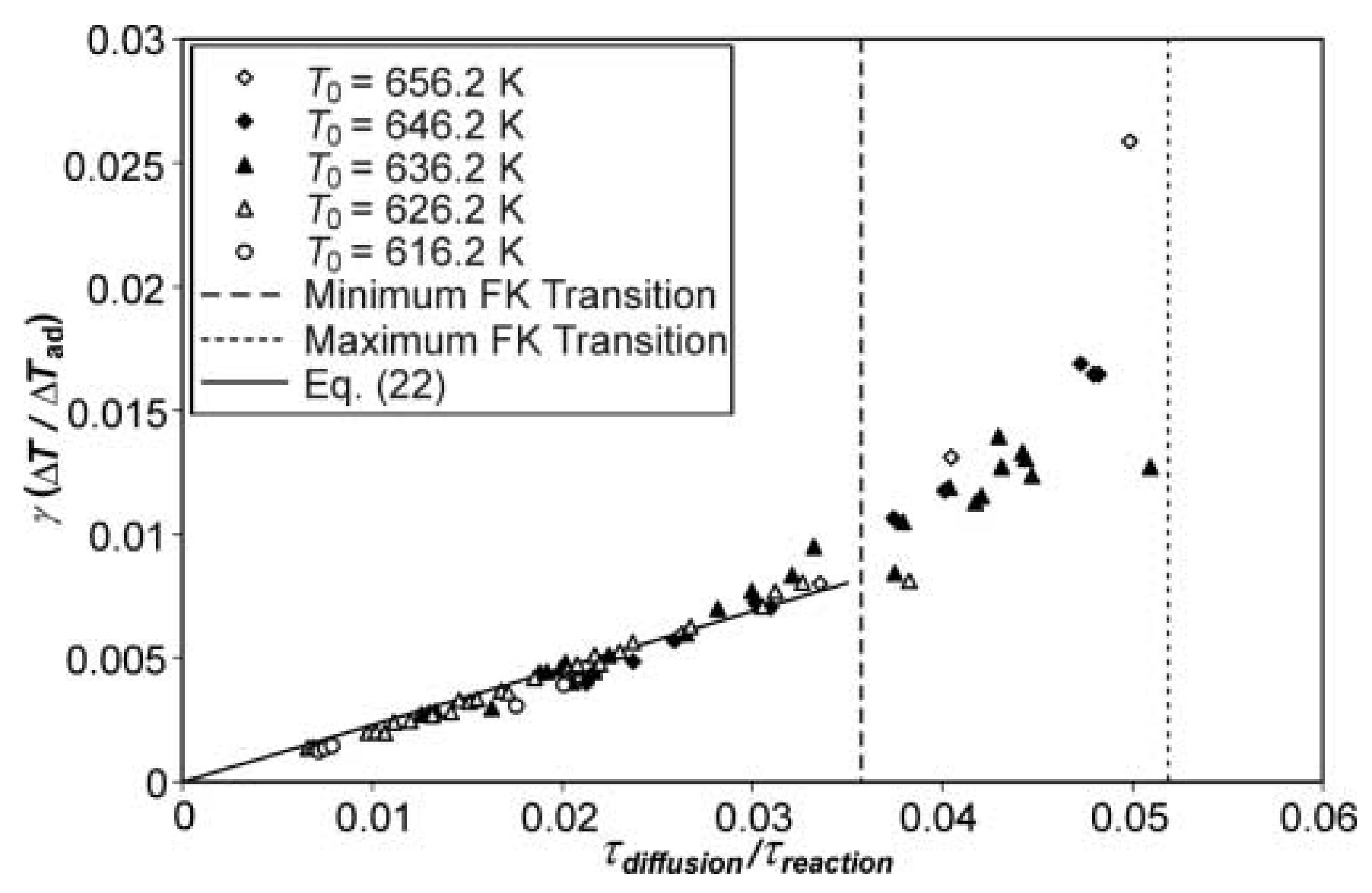


Click here to download high resolution image

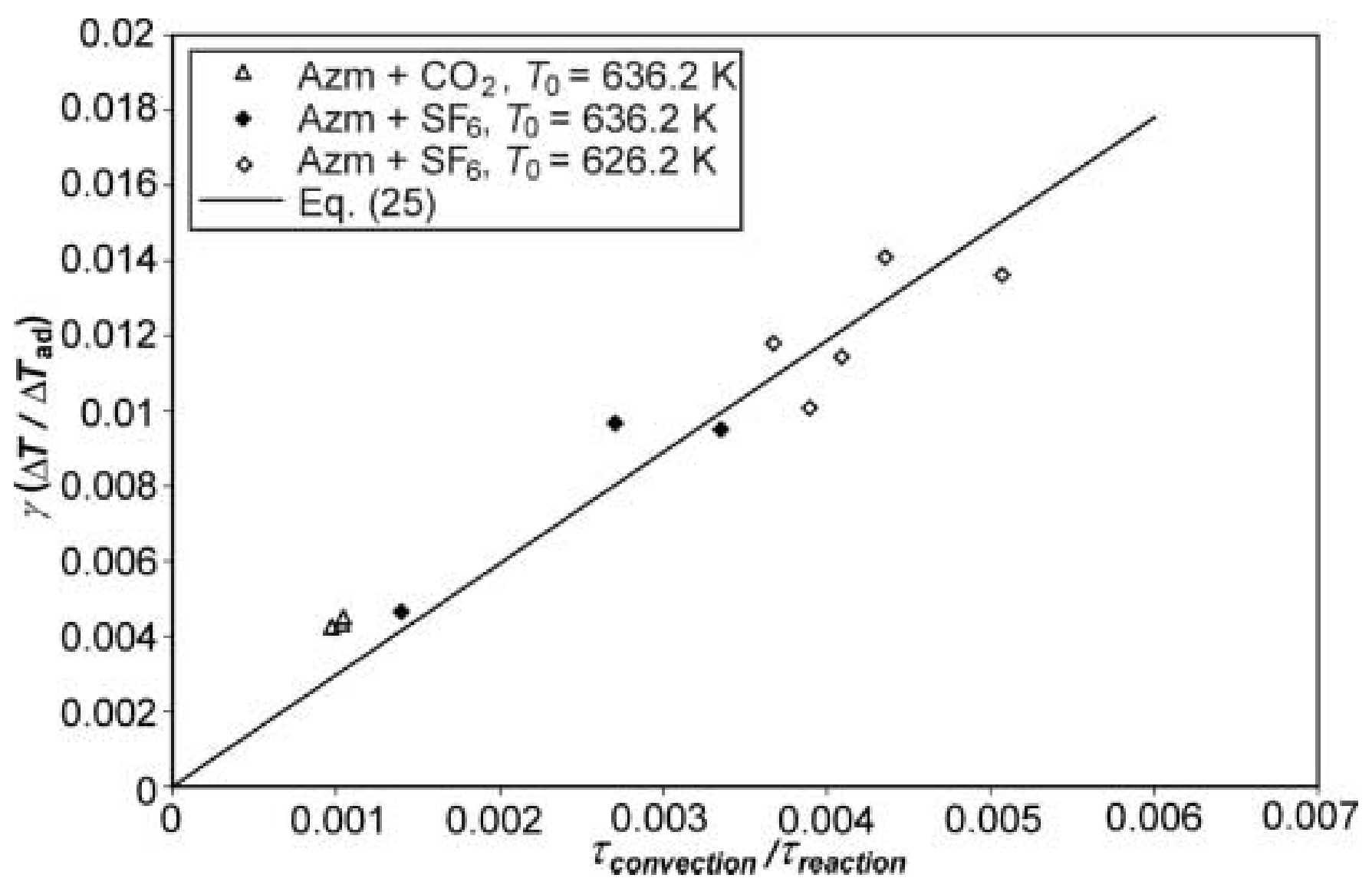


Figure 4
Click he

Click here to download high resolution image

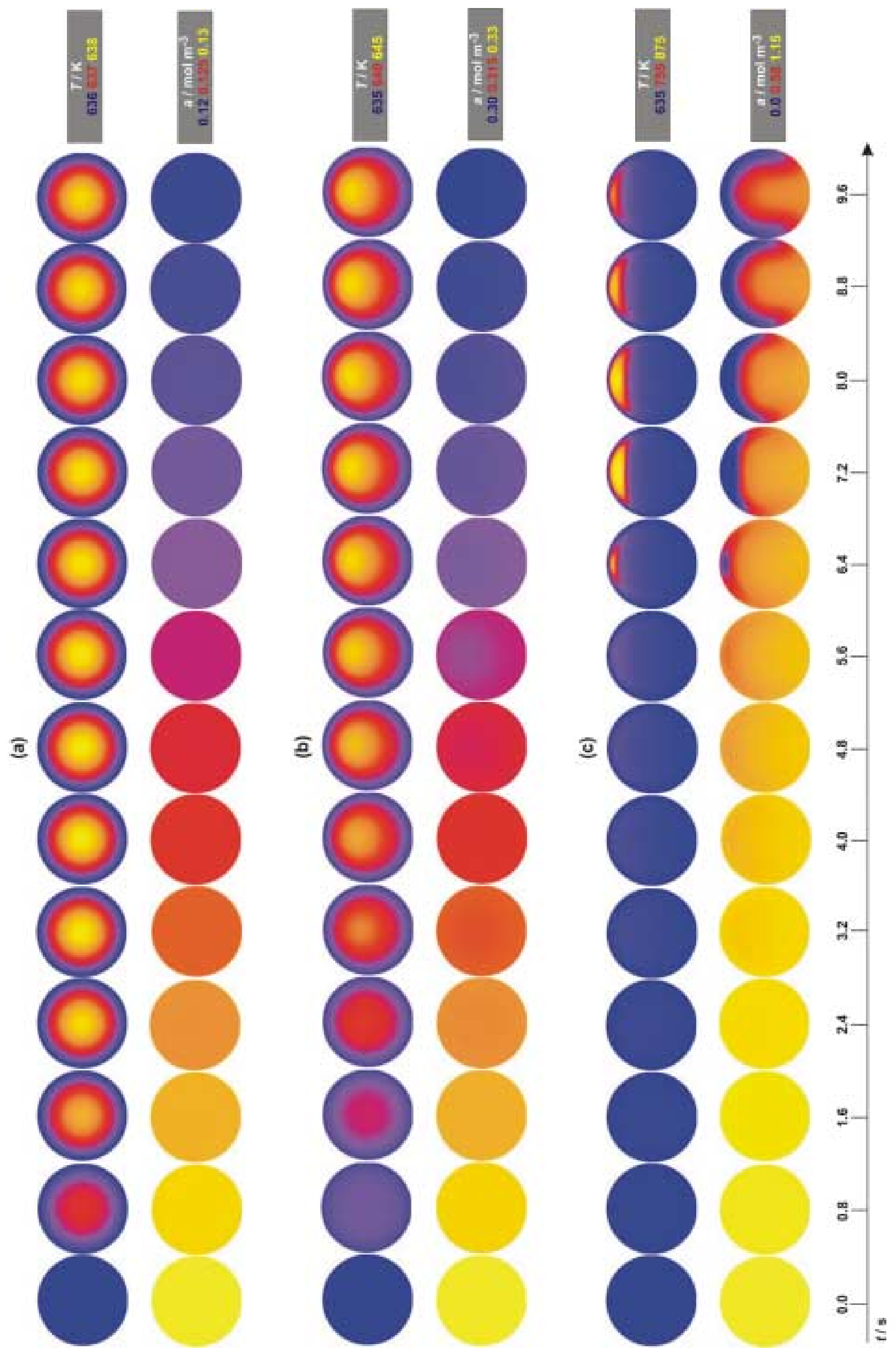



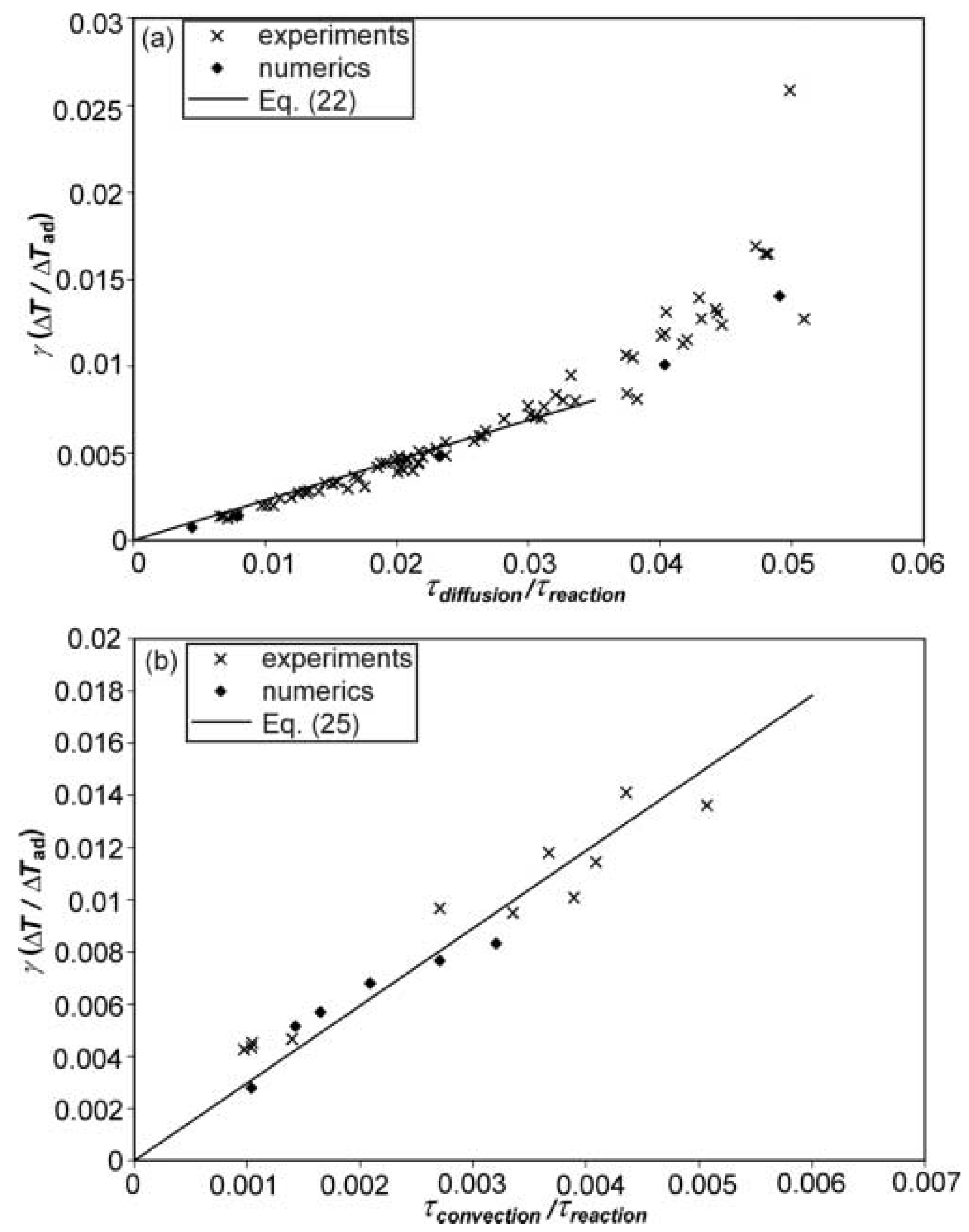
Figure 6
Click here to download high resolution image

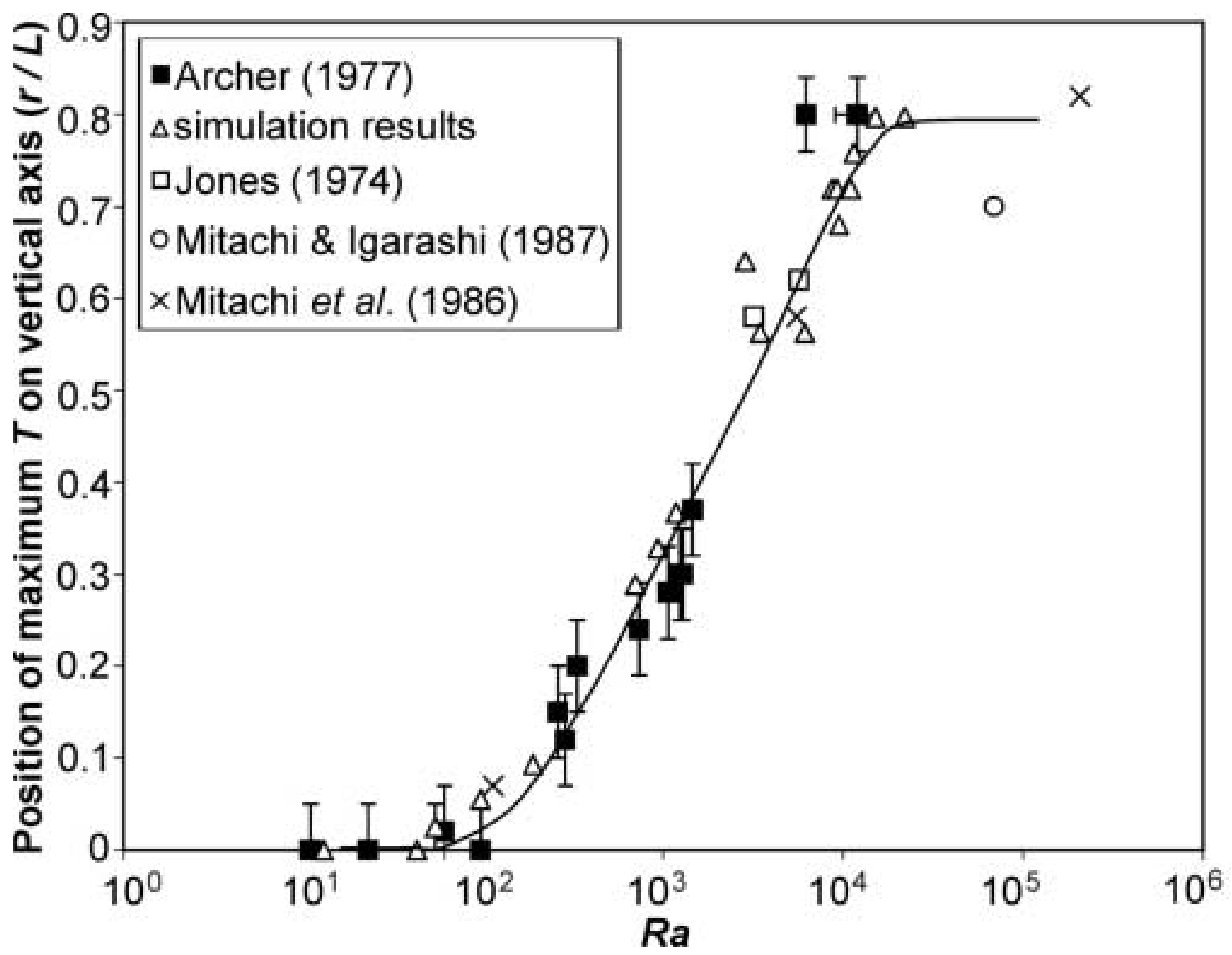


Figure 7
Click here to download high resolution image
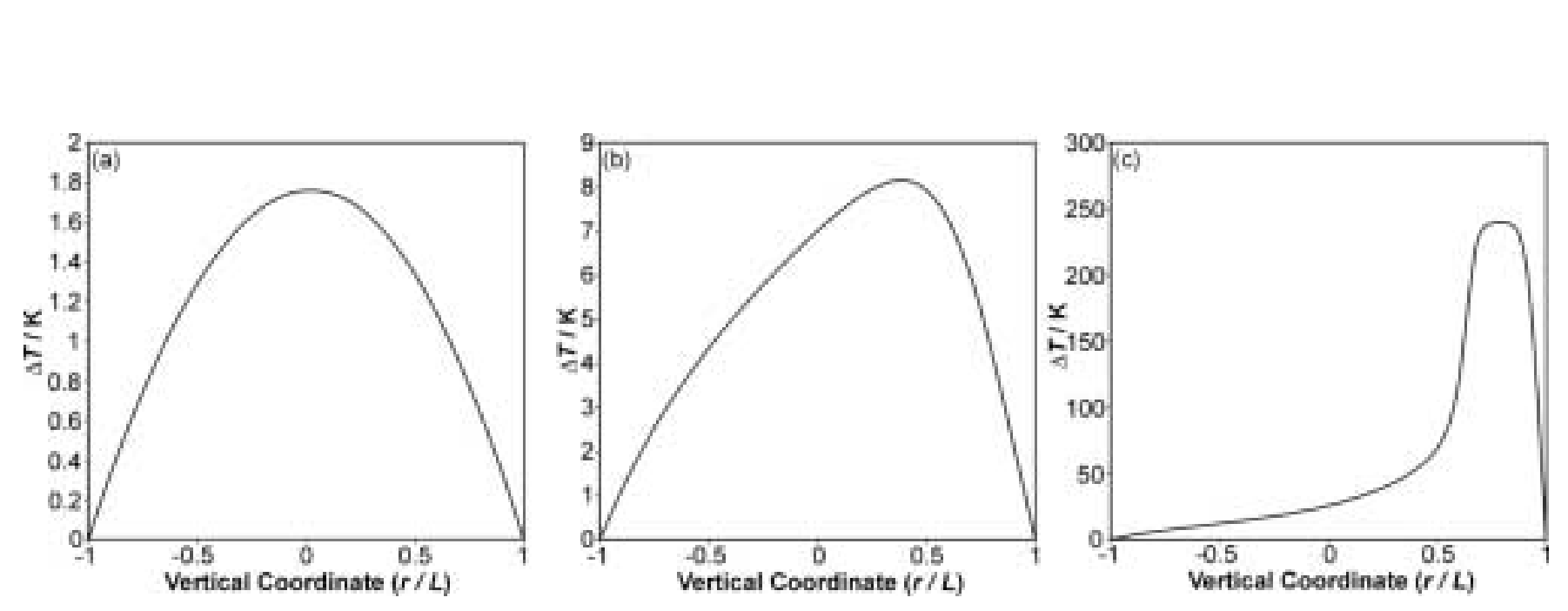

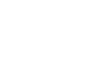

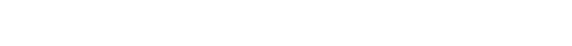

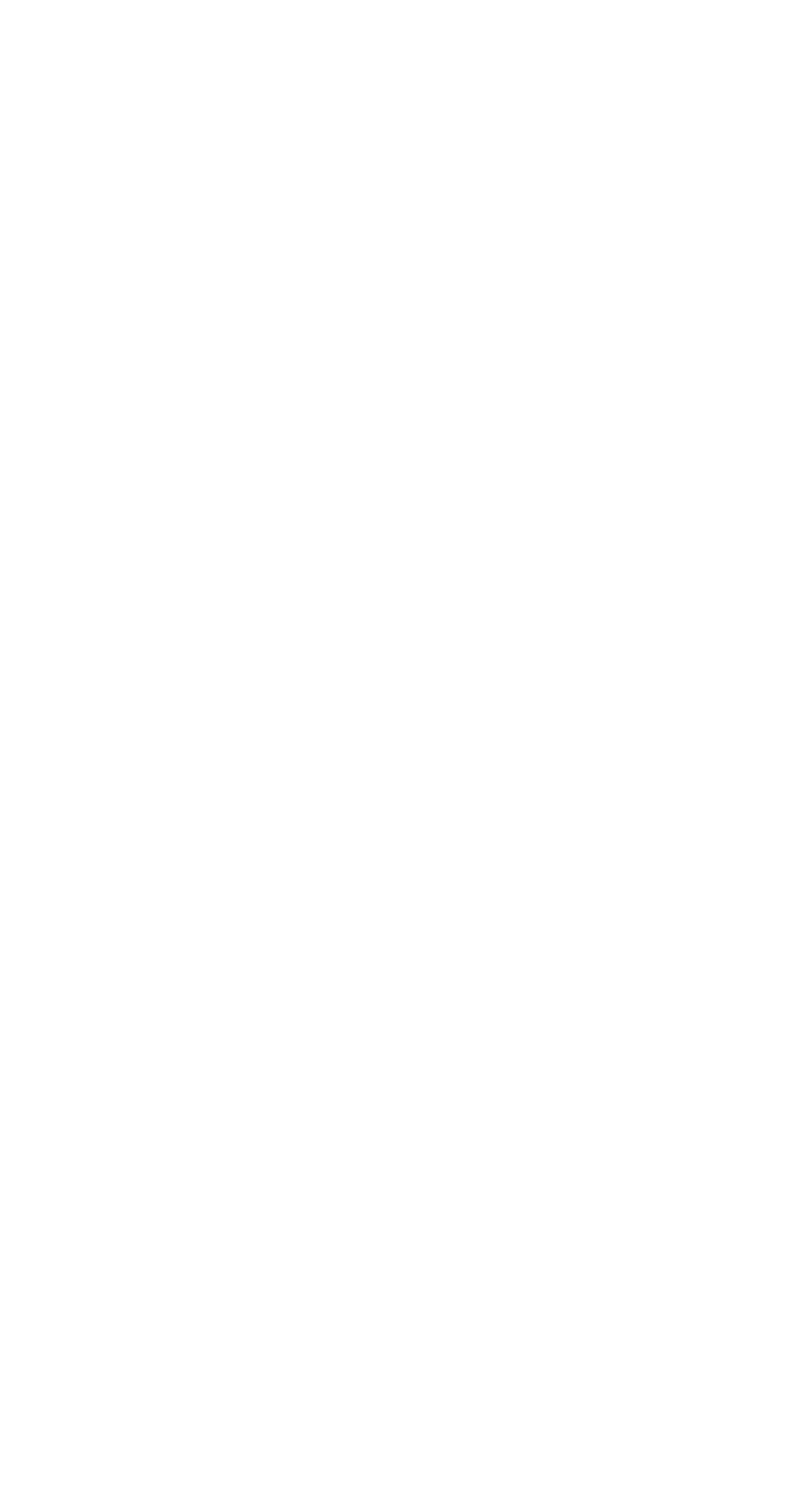

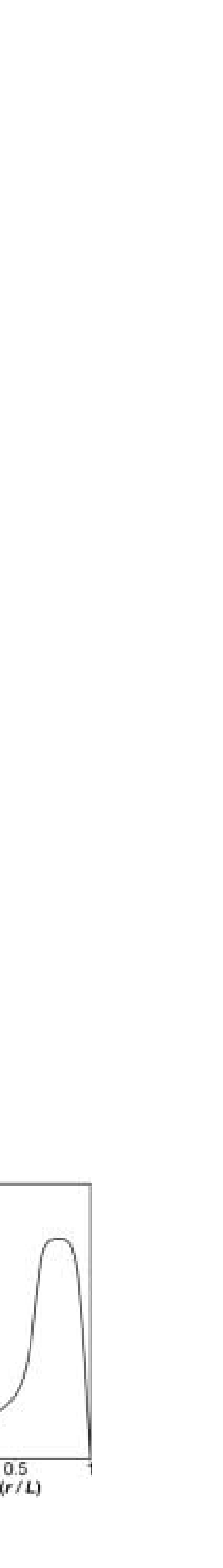


Figure 8

Click here to download high resolution image
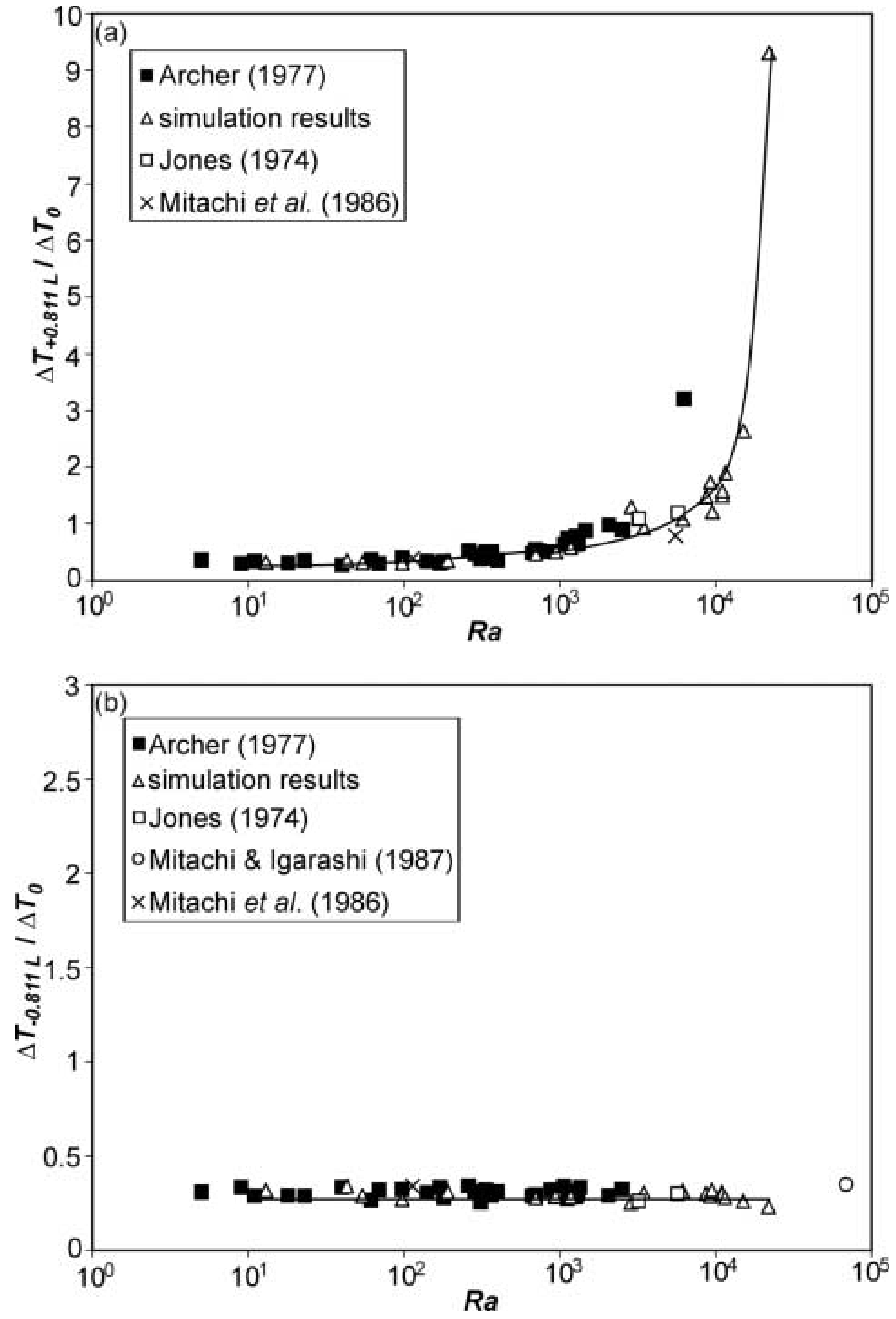
Figure 9
Click here to download high resolution image

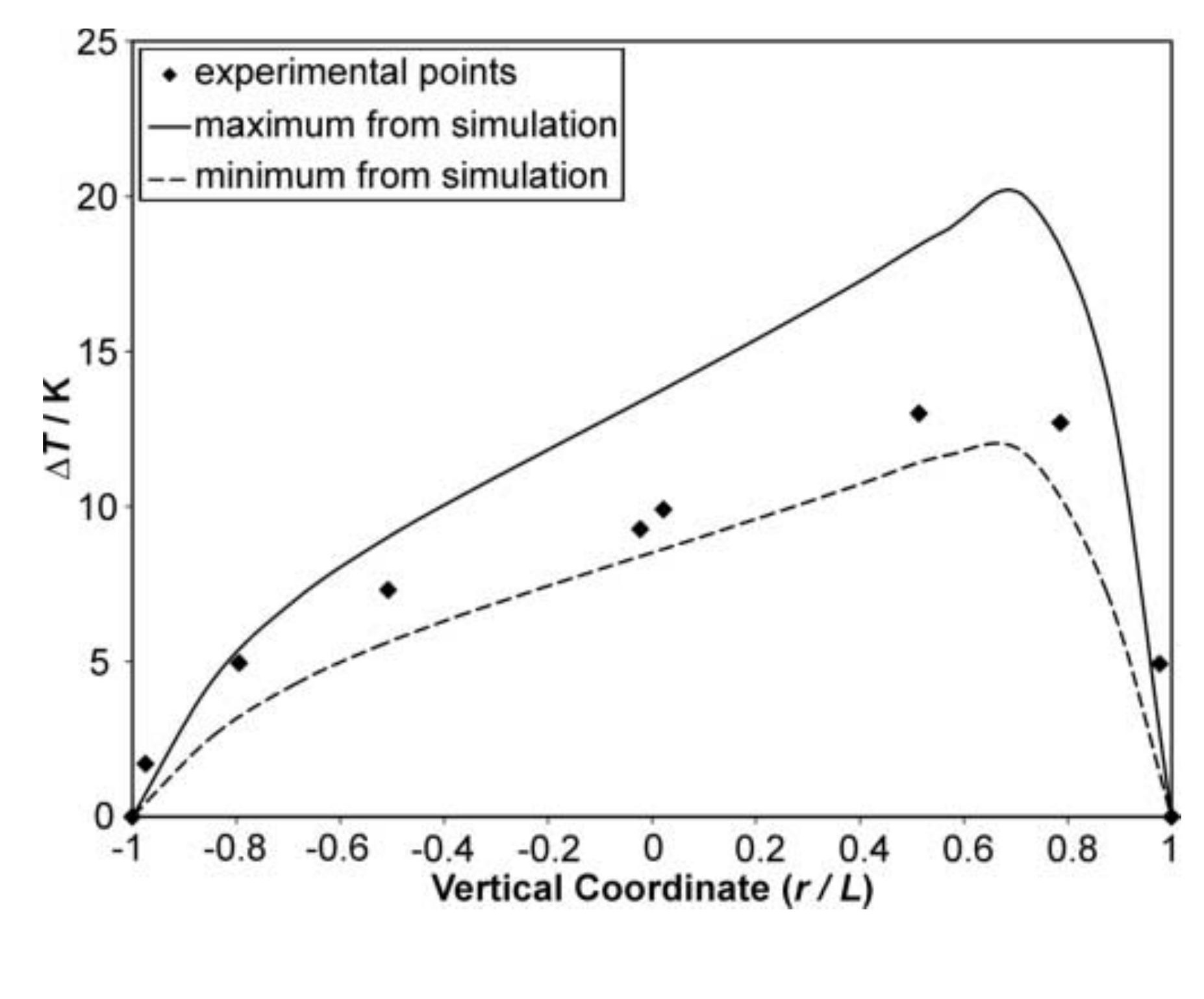

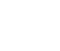

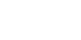


Figure 10
Click here to download high resolution image

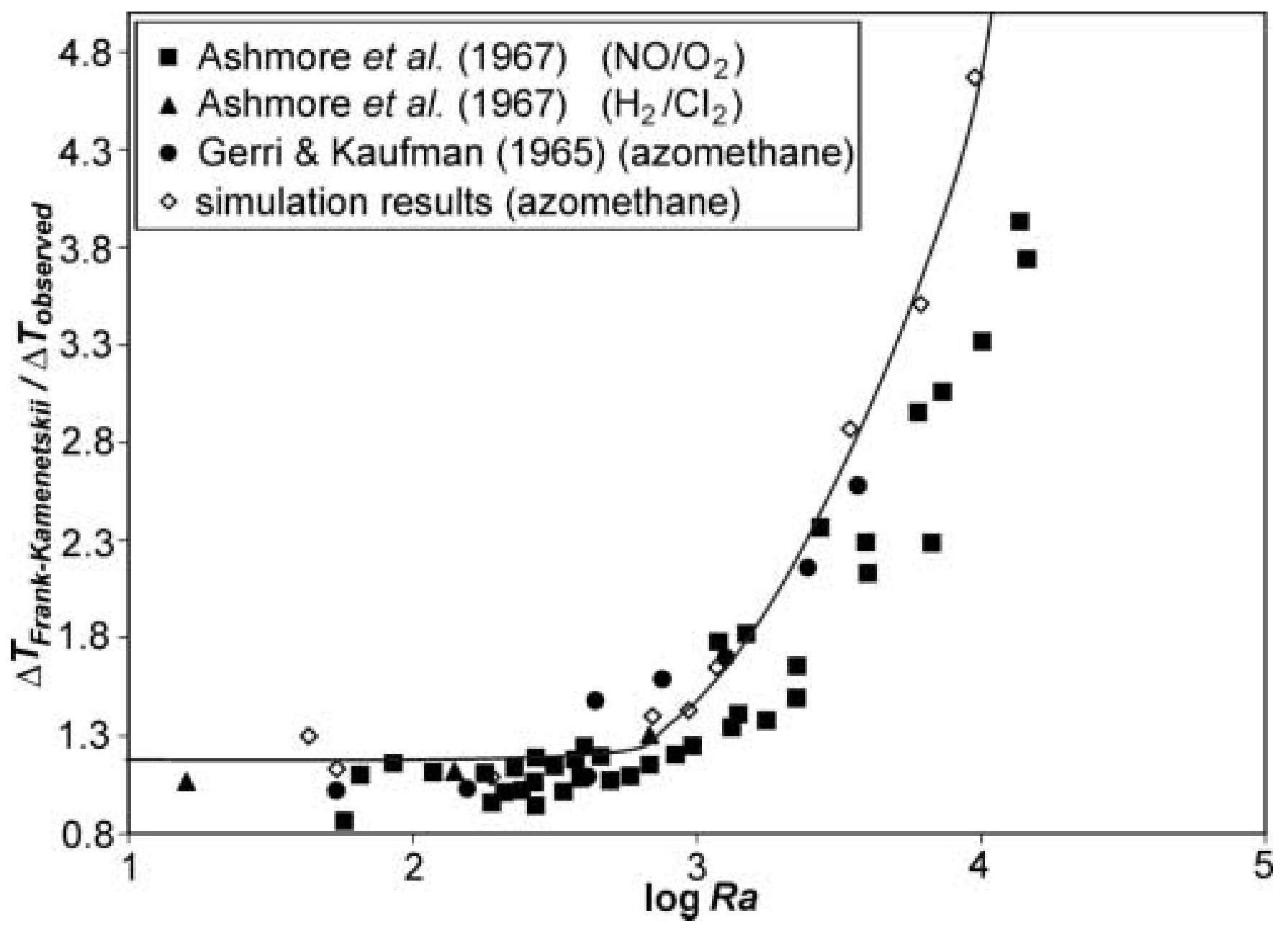

